\title{
Study of star-forming galaxies in SDSS up to redshift 0.4
}

\section{Evolution from the fundamental parameters: mass, metallicity and star formation rate}

\author{
M. A. Lara-López ${ }^{1,2}$, A. Bongiovanni ${ }^{1,2}$, J. Cepa ${ }^{1,2}$, A. M. Pérez García ${ }^{1,2}$, M. Sánchez-Portal ${ }^{4}$, H. O. Castañeda ${ }^{1,3}$, \\ M. Fernández Lorenzo ${ }^{1,2}$, and M. Povicín ${ }^{1,2}$ \\ 1 Instituto de Astrofísica de Canarias, 38200 La Laguna, Spain \\ e-mail: mall@iac.es \\ 2 Departamento de Astrofísica, Universidad de la Laguna, Spain \\ 3 Departamento de Física, Escuela Superior de Física y Matemática, IPN, México D.F., Mexico \\ ${ }^{4}$ Herschel Science Center, INSA/ESAC, Madrid, Spain
}

Received 16 December 2009 / Accepted 24 March 2010

\section{ABSTRACT}

\begin{abstract}
Context. To understand the formation and evolution of galaxies, it is important to have a full comprehension of the role played by metallicity, star formation rate (SFR), morphology, and color. The interplay of these parameters at different redshifts will substantially affect the evolution of galaxies and, as a consequence, their evolution provides important clues and constraints for the galaxy evolution models.

Aims. In this work we focus on the evolution of the SFR, metallicity of the gas, and morphology of galaxies at low redshift in search of signs of evolution. We analyzed the evolution of the SFR, metallicity, and morphology, through the mass-metallicity, luminositymetallicity, SFR-stellar mass, and SFR-metallicity relationships of star-forming galaxies from SDSS-DR5 (Sloan Digital Sky SurveyData Release 5), using redshift intervals in bins of 0.1 from $\sim 0$ to 0.4 .

Methods. We used data processed with the STARLIGHT spectral synthesis code, correcting the fluxes for dust extinction, and estimating metallicities using the $R_{23}$ method. We used the $\mathrm{S} 2 \mathrm{~N} 2(\log (\mathrm{H} \alpha /[\mathrm{S} \mathrm{II}])$ vs. $\log (\mathrm{H} \alpha /[\mathrm{N}$ II $]))$ diagnostic diagram as a tool to classify star-forming, composite, and AGN galaxies. We analyzed the evolution of the three principal BPT diagrams, estimating the SFR and specific SFR (SSFR) for our samples of galaxies, studying the luminosity and mass-metallicity relations, and analyzing the morphology of our sample of galaxies through the $g-r$ color, concentration index, and SSFR.

Results. We found that the S2N2 is a reliable diagram for classifying star-forming, composite, and AGNs galaxies. We demonstrate that the three principal BPT diagrams show an evolution toward higher values of [O III] $\lambda 5007 / \mathrm{H} \beta$ due to a metallicity decrement. We found an evolution in the mass-metallicity relation of $\sim 0.2$ dex for the redshift range $0.3<z<0.4$ compared to our local one. From the analysis of the evolution of the SFR and SSFR as a function of the stellar mass and metallicity, we discovered a group of galaxies with higher SFR and SSFR at all redshift samples, whose morphology is consistent with those of late-type galaxies. Finally, the comparison of our local $(0.04<z<0.1)$ with our higher redshift sample $(0.3<z<0.4)$ shows that the metallicity, the SFR, and morphology evolve toward lower values of metallicity, higher SFRs, and late-type morphologies for the redshift range $0.3<z<0.4$.
\end{abstract}

Key words. galaxies: abundances - galaxies: evolution - galaxies: starburst - galaxies: spiral - galaxies: star formation

\section{Introduction}

Baldwin et al. (1981, hereafter BPT) were the first to propose diagnostic diagrams for classifying galaxies into starburst or active galactic nucleus (AGN), based on the dominant energy source in emission-line galaxies, since AGNs have a much harder ionizing spectrum than hot stars. Revised and refined by Veilleux \& Osterbrock (1987), the three BPT empirical diagnostic diagrams use the optical line ratios [O I] $\lambda 6300 / \mathrm{H} \alpha,[\mathrm{S}$ II] $\lambda \lambda 6717$, $6731 / \mathrm{H} \alpha$, [N II] $\lambda 6583 / \mathrm{H} \alpha$, and [O III] $\lambda 5007 / \mathrm{H} \beta$ (hereafter $[\mathrm{N} \mathrm{II}]$ will refer to $[\mathrm{N} \mathrm{II}] \lambda 6583$, and [S II] to [S II] $\lambda \lambda 6717,6731)$. The BPT diagrams are the most widely used method of segregating between star-forming (SF) galaxies and AGNs, since the lines in SF galaxies are emitted by $\mathrm{H}$ II regions, which are ionized by massive stars, while AGNs are ionized by a harder radiation field.

Kewley et al. (2001, hereafter Kew01) used a combination of stellar population synthesis models and detailed self-consistent photoionization models to create a theoretical maximum starburst line on the three BPT diagrams. Kauffmann et al. (2003a, hereafter Kauf03) has shifted this starburst limit to a lower and more precise one in the $[\mathrm{N} \mathrm{II}] / \mathrm{H} \alpha$ diagram, excluding Seyfert-H II composite objects whose spectra contain significant contributions from both AGN and star formation, from pure SF galaxies. Galaxies between the Kauf03 and Kew01 divisions are considered as composite galaxies.

There are more division criteria between SF galaxies and AGNs in the $[\mathrm{NII}] / \mathrm{H} \alpha$ vs. [O III] $\lambda 5007 / \mathrm{H} \beta$ BPT diagram, such as the one of Stasińska et al. (2006), which used a lower limit than that of Kauf03, based on a more rigorous criterion, and the one of Lee et al. (2007a), which used an intermediate empirical line between the Kauf03 and Kew01 divisions. It is possible, however, to classify SF galaxies and AGNs using only the $[\mathrm{N} \mathrm{II}] / \mathrm{H} \alpha$ ratio, as discussed in Stasińska et al. (2006), since the left arm of the $[\mathrm{N} \mathrm{II}] / \mathrm{H} \alpha$ diagram (see for example Fig. 2) is a measure of the combination of the metallicity and the 
ionization parameter. Then, higher values of this ratio indicate that the galaxy hosts an AGN. Stasińska et al. (2006) classify as SF galaxies those with $\log ([\mathrm{N} \mathrm{II}] / \mathrm{H} \alpha) \leq-0.4$, composite galaxies those with $-0.4<\log ([\mathrm{N}$ II $] / \mathrm{H} \alpha) \leq-0.2$, and as AGNs those galaxies with $\log ([\mathrm{N} \mathrm{II}] / \mathrm{H} \alpha)>-0.2$.

Following with the objective of segregating SF from composite and AGNs galaxies, in this work we study the S2N2 diagram as a reliable segregator of galaxies. This $\log (\mathrm{H} \alpha /[\mathrm{S} \mathrm{II}])$ vs. $\log (\mathrm{H} \alpha /[\mathrm{N} \mathrm{II}])$ diagram was introduced by Sabbadin et al. (1977) as a useful tool for separating galactic planetary nebula (PNe), $\mathrm{H}$ II regions, and supernova remnants (SNRs). This diagram was later applied to Herbig-Haro objects (Cantó 1981), Galactic PNe (García-Lario et al. 1991; Riesgo \& López 2005), and extragalactic PNe (Magrini et al. 2003). The S2N2 diagram has also been used as a metallicity and ionization parameter indicator for extragalactic H II regions by Viironen et al. (2007).

The S2N2 diagram has been also applied to galaxies by some authors. For example, Moustakas \& Kennicutt (2006) studied whether there was a difference between integrated spectra of galaxies and the spectra of individual H II regions. Dopita et al. (2006) used the S2N2 diagram, among others, for abundance diagnostics using photoionization models. Nevertheless, the [S II] flux always shows deficiences when generated by photoionization models (e.g. Levesque et al. 2010). Also, Lamareille et al. (2009) and Pérez-Montero et al. (2009) used the S2N2 diagram as a segregator of SF from Seyfert 2 galaxies, but using different ratios: $\log ([\mathrm{N} \mathrm{II}] / \mathrm{H} \alpha)$ vs. $\log ([\mathrm{S} \mathrm{II}] / \mathrm{H} \alpha)$. However, in their division, Lamareille et al. (2009) do not distinguish between SF and composite galaxies; also, they use equivalent widths instead of emission line fluxes, which could affect the results (Kobulnicky \& Kewley 2004).

The formation and evolution of galaxies at different cosmological epochs are driven mainly by two linked processes: the star formation history and the metal enrichment. Thus, from an observational point of view, the star formation rate (SFR), the metallicity, and the stellar mass of the galaxies at different epochs will give us important clues to the evolution of galaxies. The first quantitative SFRs were derived from evolutionary synthesis models of galaxy colors (Tinsley 1968, 1972; Searle et al. 1973), confirming the trends in SFRs and star formation histories along the Hubble sequence and giving the first predictions of the evolution of the SFR with cosmic lookback time. The development of more precise direct SFR diagnostics includes the integrated emission-line fluxes (Cohen 1976; Kennicutt 1983), near-ultraviolet continuum fluxes (Donas \& Deharveng 1984), and infrared continuum fluxes (Harper \& Low 1973; Rieke \& Lebofsky 1978; Telesco \& Harper 1980); see Kennicutt (1998) for a review. The hydrogen Balmer line $\mathrm{H} \alpha$ is currently the most reliable tracer of star formation, since in H II regions and starforming galaxies, the Balmer emission-line luminosity scales directly with the total ionizing flux of the embedded stars. A widely known calibration of the $\mathrm{H} \alpha$ line as SFR tracer is the one devised by Kennicutt (1998). However, it is important to take corrections for stellar absorption and reddening into account to obtain SFRs in agreement with the ones derived using other wavelengths (e.g. Rosa-González et al. 2002; Charlot et al. 2002; Dopita et al. 2002). In parallel, other diagnostics have been developed using the oxygen doublet [O II] $\lambda 3726,3729$ for the redshift range $z \sim 0.4-1.5$ (e.g. Gallagher et al. 1989; Kennicutt 1998; Rosa-González et al. 2002; Kewley et al. 2004). Moreover, this diagnostic is useful when the $\mathrm{H} \alpha$ line is not easily observed at higher redshifts $(z \gtrsim 0.4$ in the optical). However, the [O II] doublet presents problems in reddening and abundance dependence (Jansen et al. 2001; Charlot et al. 2002). Alternatively, it is possible to estimate the SFR from the soft X-ray luminosity, which is comparable to the one determined from the $\mathrm{H} \alpha$ luminosity (Rosa González et al. 2009; Rovilos et al. 2009).

A strong dependence on the SFR and the stellar mass and its evolution with redshift has been found, with the bulk of star formation occurring first in massive galaxies, and later in less massive systems (e.g. Guzmán et al. 1997; Brinchmann \& Ellis 2000; Juneau et al. 2005; Bauer et al. 2005; Bell et al. 2005; Pérez-Gonzalez et al. 2005; Feulner et al. 2005; Papovich et al. 2006; Caputi et al. 2006; Reddy et al. 2006; Erb et al. 2006a; Noeske et al. 2007a; Buat et al. 2008). In the local universe, several studies have illustrated a relationship between the SFR and stellar mass, identifying two populations: galaxies on a starforming sequence, and "quenched" galaxies, with little or no detectable star formation (Brinchmann et al. 2004; Salim et al. 2005; Lee 2006; Lee et al. 2007b). At higher redshift, Noeske et al. (2007a) shows the existence of a "main sequence" (MS) for SF galaxies in the SFR-stellar mass relation over the redshift range $0.2<z<1.1$. From the galaxies considered in this study, the range of $\log (\mathrm{SFR})$ remains constant to $z>1$, while the MS as a whole moves to higher SFR as $z$ increases.

Metallicity is another important property of galaxies, and its study is crucial for a deep understanding of galaxy formation and evolution, since it is related to the whole past history of the galaxy. Metallicity is a tracer of the fraction of baryonic mass already converted into stars and is sensitive to the metal losses due to stellar winds, supernovae, and active nuclei feedbacks. A detailed description of the different metallicity methods and calibrations are given in Lara-López et al. (2009a,b).

Stellar mass and metallicity are strongly correlated in SF galaxies, with massive galaxies showing higher metallicities than less massive galaxies. This relationship provides essential insight into galaxy formation and evolution. The massmetallicity $(M-Z)$ relation first observed by Lequeux et al. (1979) has been intensively studied (Skillman et al. 1989; Brodie \& Huchra 1991; Zaritsky et al. 1994; Richer \& McCall 1995; Garnett et al. 1997; Pilyugin \& Ferrini 2000, López-Sánchez 2010; López-Sánchez \& Esteban 2010, among others), and it is well established by the work of Tremonti et al. (2004, hereafter T04) for the local universe $(z \sim 0.1)$ using SDSS data. The study of the redshift evolution of the $M-Z$ relation has provided us with basic information on the cosmic evolution of star formation.

Regarding the evolution of the $M-Z$ relation for SF galaxies at $z<1$, Savaglio et al. (2005) have investigated the massmetallicity relations using galaxies at $0.4<z<1$, finding that metallicity is lower at higher redshift by $\sim 0.15$ dex. Moreover, Maier et al. (2005), Hammer et al. (2005), and Liang et al. (2006) find that emission line galaxies are poorer in metals at $z \sim 0.7$ than present-day spirals. A study of Lamareille et al. (2009) focused on the evolution of the $M-Z$ relation up to $z \sim 0.9$, suggesting that the $M-Z$ relation is flatter at higher redshifts. However, Carollo \& Lilly (2001), from emission-line ratios of 15 galaxies in a range of $0.5<z<1$, find that their metallicities appear to be remarkably similar to those of local galaxies selected with the same criteria. Also, Lilly et al. (2003) used a sample of 66 SF galaxies with $0.47<z<0.92$ to claim a smaller variation in metallicity of $\sim 0.08$ dex compared with the metallicity observed locally, showing only modest evolutionary effects (for more details about the $M-Z$ relation, see Lara-López et al. 2009b).

In a recent study, Calura et al. (2009) have demonstrated the importance of the morphology of galaxies when deriving the $M-Z$ relation since, at any redshift, elliptical galaxies present the highest stellar masses and the highest metallicities, whereas 
the irregulars are the least massive galaxies, characterized by the lowest $\mathrm{O}$ abundances.

In this paper, we consistently approach several topics, starting with introducing the S2N2 as a reliable diagram for classifying galaxies, analyzing of the metallicity evolution of galaxies in the three BPT diagrams, and, for a better understanding of the processes involved in the observed evolution of galaxies at low redshift, studying the mass, metallicity and SFR relations, such as the $M-Z$, metallicity-SFR, and mass-SFR relations. We also point out that the morphology of galaxies play an important role when deriving conclusions, since late-type galaxies will result in lower metallicity estimates and higher SFRs than early-type (Calura et al. 2009).

This paper is structured as follows. In Sect. 2 we give the data used for this study, the dust extinction correction, and the metallicity estimates for our sample of galaxies. In Sect. 3 we introduce the $\mathrm{S} 2 \mathrm{~N} 2$ as a reliable diagram for segregating SF, composite, and AGNs galaxies. In Sect. 4 we analyze the evolution of the BPT diagrams. In Sect. 5 we investigate the evolution of the mass-metallicity and luminosity-metallicity relations, whereas in Sect. 6 we discuss the relations between the SFR and SSFR with stellar mass and metallicity, as well as the morphology of our galaxies using colors, concentration index, and SSFRs. Finally, conclusions are given in Sect. 7.

\section{Data processing and sample selection}

We selected emission line galaxies from SDSS-DR5 (Adelman-McCarthy et al. 2007). Data were taken with a $2.5 \mathrm{~m}$ telescope located at Apache Point Observatory (Gunn et al. 2006). The SDSS spectra were obtained using 3 arcsec diameter fibres, covering a wavelength range of 3800-9200 $\AA$, and with a mean spectral resolution $\lambda / \Delta \lambda \sim 1800$. The SDSSDR5 spectroscopy database contains spectra for $\sim 10^{6}$ objects over $\sim 5700 \mathrm{deg}^{2}$. Further technical details can be found in Stoughton et al. (2002).

We used the SDSS-DR5 spectra from the STARLIGHT database $^{1}$, which were processed with the STARLIGHT spectral synthesis code, developed by Cid Fernandes and collaborators (Cid Fernandes et al. 2005, 2007; Mateus et al. 2006; Asari et al. 2007). From the spectra, the STARLIGHT code subtracts the continuum, obtaining the emission lines flux measurements for each galaxy. For each emission line, the STARLIGHT code returns the rest frame flux and its associated equivalent width, linewidth, velocity displacement relative to the rest-frame wavelength, and the signal-to-noise ratio $(\mathrm{S} / \mathrm{N})$ of the fit. For Balmer lines, the underlying stellar absorption was corrected by the STARLIGHT code using synthetic spectra obtained by fitting an observed spectrum with a combination of 150 simple stellar populations (SSPs) from the evolutionary synthesis models of Bruzual \& Charlot (2003).

From the full set of galaxies, we only consider galaxies whose spectra show the $\mathrm{H} \alpha, \mathrm{H} \beta$, [N II], [O II] $\lambda 3727$, [O III] $\lambda 4959$, [O III] $\lambda 5007,[\mathrm{O}$ I] $\lambda 6300$, and [S II] lines in emission. We selected galaxies with a $\mathrm{S} / \mathrm{N}$ higher than $3 \sigma$ for the $\mathrm{H} \alpha, \mathrm{H} \beta$, and $[\mathrm{N} \mathrm{II}]$ lines.

To identify any evolution of galaxy parameters or relations, we divided our sample in four redshift intervals as follows: $0.04 \leq z_{0}<0.1,0.1 \leq z_{1}<0.2,0.2 \leq z_{2}<0.3,0.3 \leq z_{3} \leq 0.4$. The lower limit of $z_{0}$ corresponds to an aperture covering fraction of $20 \%$, which is the minimum required to avoid domination of the spectrum by aperture effects (Kewley et al. 2005). This

${ }^{1}$ http://www.starlight.ufsc.br

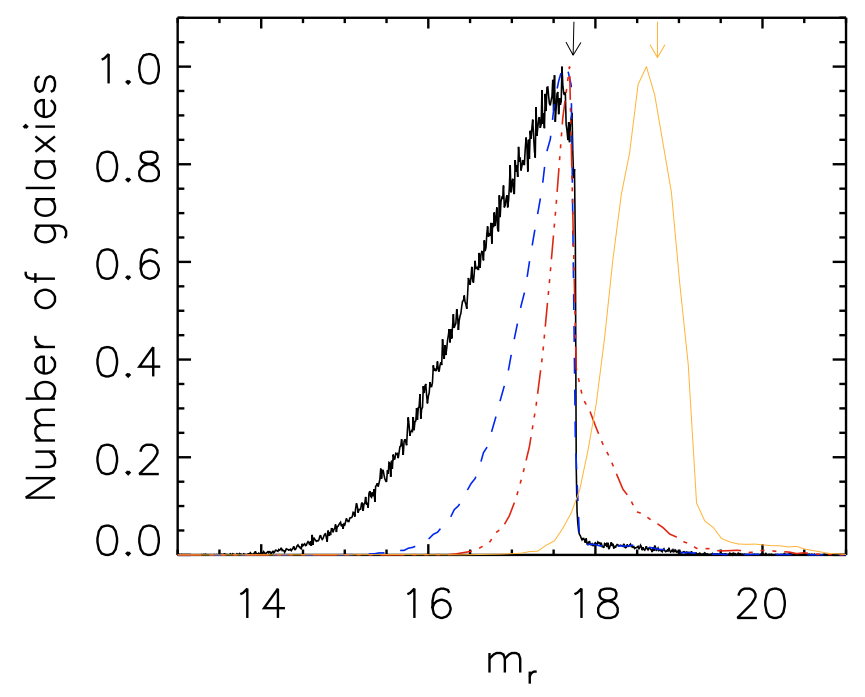

Fig. 1. Normalized histogram of the apparent Petrosian $r$ magnitudes in the four redshift bins. The dark solid line represents galaxies at $z_{0}$, the dashed line the galaxies at $z_{1}$, dot-dashed lines the galaxies at $z_{2}$, and the clear solid line the galaxies at $z_{3}$. The black arrow shows the completeness limit for the samples $z_{0}, z_{1}$, and $z_{2}$, and the clear arrow points to the same for $z_{3}$. (See the electronic edition of the Journal for a color version of this figure.)

classification give us 85931 galaxies for $z_{0}, 48888$ galaxies for $z_{1}, 3278$ galaxies for $z_{2}$, and 199 galaxies for $z_{3}$.

We selected galaxies with an apparent Petrosian $r$ magnitude of $14.5<r<17.77$ in the redshift samples $z_{0}, z_{1}$, and $z_{2}$, which yields 82884,44763 , and 1802 galaxies, respectively, corresponding to the magnitude completeness at these redshifts (see Fig. 1). Galaxies of the $z_{3}$ sample have a different completeness range $16.9<r<18.8$, as observed in Fig. 1, giving 119 galaxies. We used the $z_{0}$ and $z_{1}$ sample of galaxies with its respective completeness, but for galaxies of samples $z_{2}$ and $z_{3}$ we used both those in the completeness range and those out of the completeness range. The reason for this was to improve the galaxy statistics by increasing their number. As we show in the next sections, the main results are similar using galaxies in the magnitude completeness and galaxies of the total sample.

\subsection{Sample selection for Sect. 3}

In Sect. 3, to study the S2N2 diagram as a segregator of different types of galaxies, we adopt the "main galaxy sample" (e.g. Strauss et al. 2002) with Petrosian $r$ magnitudes in the range $14.5<r<17.77$, and the redshift interval $z_{0}$, taking all the emission lines into account and the $\mathrm{S} / \mathrm{N}$ mentioned above, which yields 82884 galaxies. In this section, we used AGN, composite, and SF galaxies.

\subsection{Sample selection for Sect. 4}

In Sect. 4, we study the evolution of the three BPT diagrams using the four redshift intervals, magnitude intervals, and the $\mathrm{S} / \mathrm{N}$ restrictions mentioned above. In this section, we used AGN, composite, and SF galaxies. Also, to study the metallicity evolution of the SF galaxies of the BPT diagrams (see Fig. 6), we used the sample of Sects. 5 and 6, mentioned below. 


\subsection{Sample selection for Sects. 5 and 6}

For Sects. 5 and 6, we selected SF galaxies following the criterion given by Kauf03 in the BPT empirical diagnostic diagram: $\log [\mathrm{O}$ III $] \lambda 5007 / \mathrm{H} \beta \leq 0.61 /\{\log ([\mathrm{N} \mathrm{II}] / \mathrm{H} \alpha)-0.05\}+1.3$, the same used by Veilleux \& Osterbrock (1987), Kewley et al. (2001, 2006), and Stasińska et al. (2006), among others. After all these selections, the number of galaxies of each redshift bin is reduced to $61921 \mathrm{SF}$ galaxies for $z_{0}, 27853$ for $z_{1}, 1671$ for $z_{2}$, and $67 \mathrm{H}$ II galaxies for $z_{3}$.

The extinction correction and metallicity estimates were calculated as in Lara-López et al. (2009b). Our extinction correction was derived using the Balmer decrements in order to obtain the reddening coefficient $\mathrm{C}(\mathrm{H} \beta)$. We used the Cardelli et al. (1989) law, with $R_{v}=A_{v} / E(B-V)=3.1$, assuming case B recombination with a density of $100 \mathrm{~cm}^{-3}$ and a temperature of $10^{4} \mathrm{~K}$, with $\mathrm{H} \alpha / \mathrm{H} \beta=2.86$ (Osterbrock 1989).

We estimated metallicities using the $R_{23}$ relation introduced by Pagel et al. (1979), $R_{23}=([\mathrm{O}$ II $] \lambda 3727+[\mathrm{O}$ III $]$ $\lambda \lambda 4959,5007) / \mathrm{H} \beta$, and adopted the calibration given by Tremonti et al. (2004), $12+\log (\mathrm{O} / \mathrm{H})=9.185-0.313 x-$ $0.264 x^{2}-0.321 x^{3}$, where $x=\log R_{23}$. We selected the upper branch of the double-valued $R_{23}$, in which the Tremonti et al. (2004) calibration is valid, taking $12+\log (\mathrm{O} / \mathrm{H})>8.4$ and $\log ([\mathrm{N} \mathrm{II}] /[\mathrm{O} \mathrm{II}] \lambda 3727)>-1.2$, since the upper and lower branches of the $R_{23}$ calibration bifurcates at those values (see Kewley \& Ellison 2008).

After applying this final criterion, we end with 58866 galaxies for $z_{0}, 24385$ for $z_{1}, 1631$ for $z_{2}$ (from which 712 galaxies are in their completeness magnitude interval), and 62 galaxies for $z_{3}$ (from which 41 galaxies are in their completeness magnitude interval), all of them in the upper branch of the $R_{23}$ relation, corresponding to the $\sim 99 \%$ to the H II classified galaxies. Then, we are not introducing a bias when selecting the upper branch for this samples.

\section{The S2N2 diagnostic diagram as a star-forming, composite, and AGN galaxy segregator}

As mentioned in Sect. 1, BPT diagrams are the most used method of segregating star-forming and AGN galaxies. From the three BPT diagrams $([\mathrm{N} \mathrm{II}] / \mathrm{H} \alpha,[\mathrm{S} \mathrm{II}] / \mathrm{H} \alpha$, and [O I] $\lambda 6300 / \mathrm{H} \alpha$ vs. [O III] $\lambda 5007 / \mathrm{H} \beta$ ), the most used one is the [N II] $/ \mathrm{H} \alpha$ vs. [O III] $\lambda 5007 / \mathrm{H} \beta$, since it is the only one that can segregate pure SF and composite galaxies, as demonstrated by Kewley et al. (2006) and Pérez-Montero et al. (2009). The two other BPT diagrams are not useful for segregating SF from composite objects.

Commonly known as the $\mathrm{S} 2 \mathrm{~N} 2$, the $\log (\mathrm{H} \alpha /[\mathrm{S} \mathrm{II}])$ vs. $\log (\mathrm{H} \alpha /[\mathrm{N} \mathrm{II}])$ diagram has been used to separate planetary nebulae (PNe), H II regions, and supernova remnants (SNRs, see Sabbadin et al. 1977; Riesgo \& López 2006; Viironen et al. 2007). We propose the S2N2 diagram for classifying SF, composite, and AGN galaxies, something until now only possible with the $[\mathrm{N} \mathrm{II}] / \mathrm{H} \alpha$ vs. [O III] $\lambda 5007 / \mathrm{H} \beta$ diagram. However, the $\mathrm{S} 2 \mathrm{~N} 2$ diagram use only the $\mathrm{H} \alpha,[\mathrm{N} \mathrm{II}]$, and [S II] emission lines, all of them close in wavelength, avoiding reddening corrections, and making possible its use for surveys limited in the spectral range.

From the "main galaxy sample", we consider SF galaxies as those lying below the Kauf03 division, composite galaxies as those lying between the Kauf03 and Kew01 lines, and AGN galaxies those above the Kew01 division (see Fig. 2). From the total sample (82 884 galaxies, see Sect. 2), the $71.4 \%$,

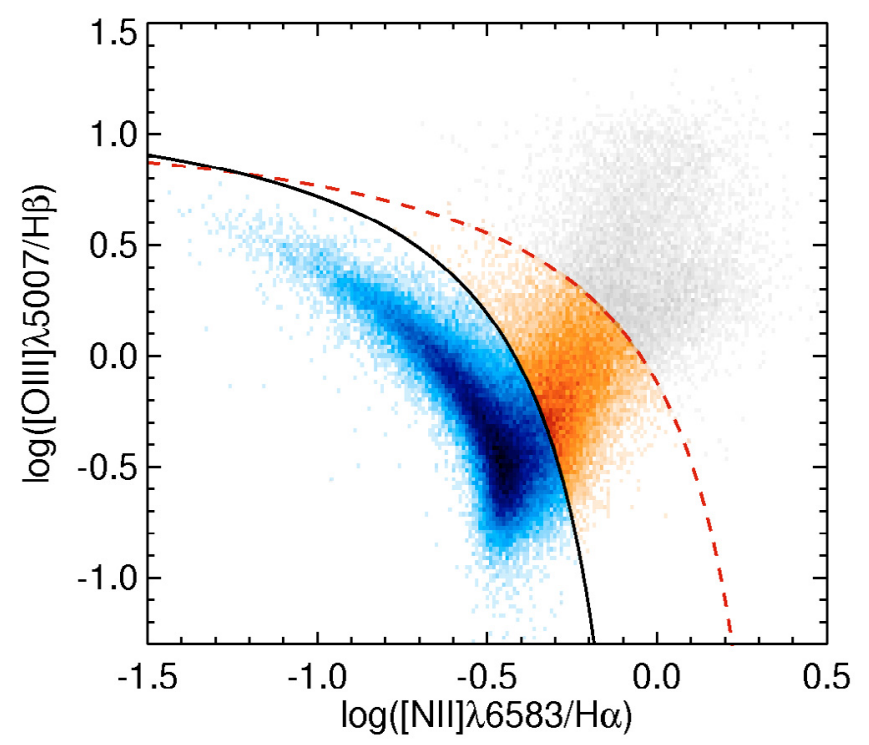

Fig. 2. $\log ([\mathrm{N} \mathrm{II}] / \mathrm{H} \alpha)$ vs. $\log ([\mathrm{O} I I I] \lambda 5007 / \mathrm{H} \beta)$ BPT diagram. The solid line shows the Kauf03 empirical division between SF and composite galaxies, and the dashed line represents the Kew01 starburst limit. (See the electronic edition of the Journal for a color version of this figure.)

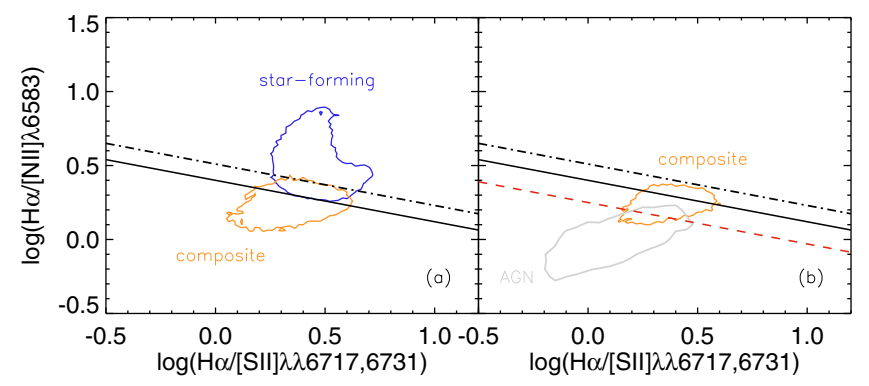

Fig. 3. Contour plots for star-forming, composite, and AGN galaxies. a) Star-forming, darker (blue) contour, and composite, lighter (orange) contour, enclosing the $\sim 90 \%(\sim 1.8 \sigma)$ of each sample. The solid line establishes our limit for star-forming galaxies, while the dot-dashed line delimits the almost pure star-forming galaxies. b) Composite, darker (orange) contour, and AGN, lighter (gray) contour, enclosing $\sim 75 \%$ $(\sim 1.2 \sigma)$ of each sample. The dashed line shows our separation for composite and AGN galaxies, with solid and dot-dashed lines as in panel a). (See the electronic edition of the Journal for a color version of this figure.)

$19 \%$, and $9.6 \%$ correspond to SF, composite, and AGN galaxies, respectively. Taking this classification as a reference, we plotted the S2N2 diagram with the three classifications of galaxies (see Figs. 3 and 4).

To establish division lines to separate SF, composite and AGN galaxies in the S2N2 diagram, we generated contour plots for each category of galaxies (see Fig. 3). Because our sample of galaxies is larger for the SF and composite galaxies, we used contours enclosing $\sim 90 \%$ for those galaxies. However, as AGN galaxies are less numerous, we used contours enclosing $\sim 75 \%$ for composite and AGNs. The contour plots shown in Fig. 3a delimit two tangent parallel lines, generating with this criterium division lines defined by Eqs. (1) and (2). To define a division line between composite and AGN galaxies, we sampled the plot area with parallel lines of Eq. (1) in bins of 0.02 dex, in this way generating histograms for composite and 
M. A. Lara-López et al.: Evolution from fundamental parameters in SDSS galaxies. II.
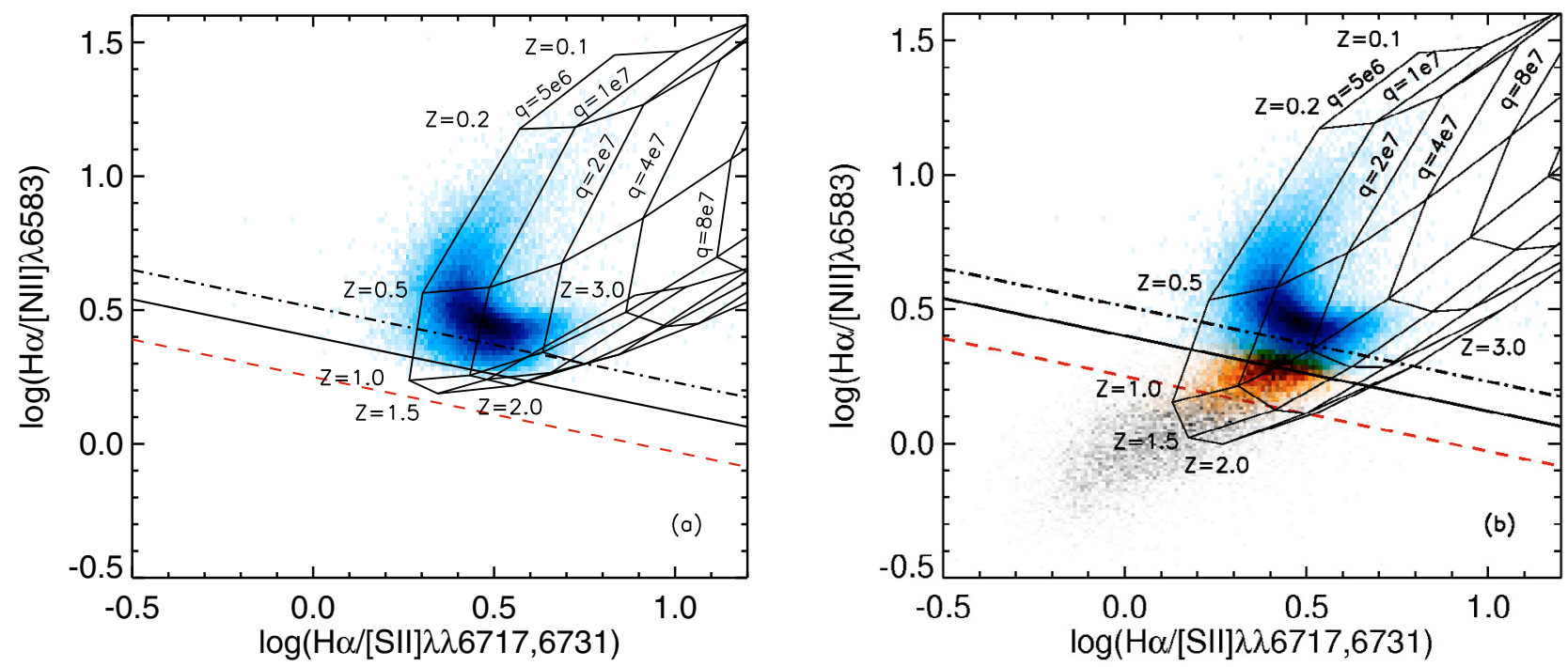

Fig. 4. a) $\log (\mathrm{H} \alpha /[\mathrm{S} \mathrm{II}])$ vs. $\log (\mathrm{H} \alpha /[\mathrm{N}$ II $])$ diagram, corresponding to the star-forming galaxies below the Kauf03 separation in the BPT diagram. We overplotted the grid of the photoionization models of Kewley et al. (2001) created with PEGASE, taking an instantaneous burst model with an electronic density of $10 \mathrm{~cm}^{-3}$. Dot-dashed, solid, and dashed lines represent our division for pure star-forming and composite, star-forming and composite, and composite and AGN galaxies, respectively. b) Same diagram with the grid of the photoionization models of Kewley et al. (2001) created with PEGASE, taking a continuous model with an electronic density of $10 \mathrm{~cm}^{-3}$. (See the electronic edition of the Journal for a color version of this figure.)

AGN galaxies, where Eq. (3) corresponds to the intersection of both histograms.

(i) Pure star-forming galaxies are separated by

$\log (\mathrm{H} \alpha /[\mathrm{N} \mathrm{II}])>-0.28 \times \log (\mathrm{H} \alpha /[\mathrm{S} \mathrm{II}])+0.51$,

and $98.8 \%$ of galaxies above this line are SF galaxies and correspond to $88 \%$ of the SF sample.

(ii) Star-forming and composite galaxies are divided by

$\log (\mathrm{H} \alpha /[\mathrm{N} \mathrm{II}])>-0.28 \times \log (\mathrm{H} \alpha /[\mathrm{S} \mathrm{II}])+0.40$,

where $99.8 \%$ of the SF sample lay above this line. However, from all galaxies above this line, $8 \%$ will correspond to composite galaxies, and the remaining $91 \%$ to SF galaxies.

(iii) Composite and AGN galaxies are divided by

$\log (\mathrm{H} \alpha /[\mathrm{N} \mathrm{II}])>-0.28 \times \log (\mathrm{H} \alpha /[\mathrm{S} \mathrm{II}])+0.25$,

with $74.6 \%$ of the galaxies below this line are AGNs. Unfortunately, this diagram does not allow separating Seyfert from Liner galaxies (see Fig. 3). Composite galaxies are selected as those between the lines of Eqs. (1) and (3). From all galaxies between both lines, $61.2 \%, 33.2 \%$, and $5.6 \%$ correspond to composite, SF, and AGN galaxies, respectively.

In Table 1 we compare the $\mathrm{N} 2$ ratio, considering the Stasińska limits, with the S2N2 diagram in order to test their respective ability for segregating SF, composite, and AGN galaxies. This table shows the percentage of galaxies classified according to each division line, as well as its corresponding contamination and the percentage of missed galaxies, taking as a reference the classification of the $\log ([\mathrm{N} \mathrm{II}] / \mathrm{H} \alpha)$ vs. $\log ([\mathrm{O} \mathrm{III}] \lambda 5007 / \mathrm{H} \beta)$ diagram. Labels S2N2(1\&3) and S2N2(2) refer to galaxies taking as a reference Eqs. (1\&3) and (2), respectively. The SF block indicates for the S2N2(2) division, for example, that by taking all galaxies above Eq. (2), 91\% will correspond to SF galaxies, and the $8 \%$ remaining to composite galaxies; also, we have missed $0.2 \%$ of the original SF sample. As can be appreciated for the SF segregation, the N2 division misses the highest percentage of SF galaxies, while our pure star-forming division (Eq. (1)) misses less SF galaxies, although with the same contamination from composite galaxies. Moreover, our SF limit (Eq. (2)) encloses almost all SF galaxies with a small contamination from composite galaxies. This provides the additional advantage over the $\mathrm{N} 2$ diagram, i.e., that the user can choose between the possibilities of selecting either most SF galaxies or galaxies with the least contamination from composite galaxies. The composite division for the N2 ratio shows a similar percentage of composite and SF galaxies. However, the S2N2 division allows obtaining a higher percentage of composite galaxies, missing less composite galaxies, with a lower contamination of SF galaxies and with only a quite small increment of AGN contamination. For segregating AGNs, while the S2N2 diagram provides a lower contamination from composite galaxies, miss $\sim 6 \%$ more AGNs than using the $\mathrm{N} 2$ ratio.

In Fig. 4, we overplot the pre-run photoionization grids of Dopita et al. (2000) and Kew01 for an instantaneous burst model and for a continuous starburst model. The best grid to our SF galaxies for the S2N2 diagram is the one corresponding to an instantaneous burst model, with an electronic density of $10 \mathrm{~cm}^{-3}$, using the PEGASE code (see Fig. 4a). As explained in Dopita et al. (2000), the high surface-brightness, isolated extragalactic $\mathrm{HII}$ regions are in general excited by young clusters of OB; in this case, the ionizing EUV spectra and $\mathrm{H}$ II region emission-line spectra predicted by the PEGASE and STARBURST99 codes for an instantaneous, zero-age star formation model are essentially identical.

In their work, Dopita et al. (2000) and Kew01 model a large sample of infrared starburst galaxies using both the PEGASE v2.0 (Fioc \& Rocca-Volmerange 1997) and the STARBURST99 (Leitherer et al. 1999) codes to generate the spectral energy distribution. In both cases, MAPPINGS III code was used to compute photoionization models. The pre-run grids use photoionization models with ionization parameters $q$ $\left(\mathrm{cm} \mathrm{s}^{-1}\right)$ in the range $5 \times 10^{6} \leq q \leq 3 \times 10^{8}$, and metallicities from $Z=0.05$ to $3 Z_{\odot}$; moreover, two values for electronic density were used, 10 and $350 \mathrm{~cm}^{-3}$. 
A\&A 519, A31 (2010)

Table 1. Comparison between the S2N2 diagram and the N2 ratio to segregate SF, composite, and AGN galaxies.

\begin{tabular}{|c|c|c|c|c|c|c|c|c|c|c|}
\hline & \multicolumn{3}{|c|}{ SF segregation (\%) } & \multicolumn{4}{|c|}{ Composite segregation $(\%)$} & \multicolumn{3}{|c|}{ AGN segregation (\%) } \\
\hline & SF & SF-missed & Comp & Comp & Comp-missed & SF & AGN & AGN & AGN-missed & Comp \\
\hline $\mathrm{N} 2$ & 98.8 & 18.6 & 1.2 & 49.3 & 27.3 & 47.7 & 3 & 66 & 9 & 34 \\
\hline $\mathrm{S} 2 \mathrm{~N} 2(1 \& 3)$ & 98.8 & 11.8 & 1.2 & 61.2 & 18.6 & 33.2 & 5.6 & 74.6 & 15.3 & 25.4 \\
\hline S2N2(2) & 91 & 0.2 & 8 & & & & & & & \\
\hline
\end{tabular}

For starburst galaxies, it is expected to have a continuous star formation on at least a galactic dynamical timescale, at which point the assumption of a continuous rather than an instantaneous burst of star formation would be more accurate. We generated the PEGASE grid of Kew01 for a continuous starburst model and found, as expected, that this corresponds to SF and composite objects (see Fig. 4b), since this is the limit used by Kew01 to parametrize an extreme starburst line in the BPT diagrams.

The photoionization grids generated with the STARBURST99 code were not hard enough to produce the needed [S II] flux to enclose all galaxies in the S2N2 diagram. The BPT diagrams are most sensitive to the spectral index of the ionizing radiation field in the $1-4$ ryd interval, and the PEGASE ionizing stellar continuum is harder in this range than that of STARBURST99, therefore PEGASE are the only models that encompass nearly all of the observed starburst on all three of the BPT diagrams. We also tried the grids of Levesque et al. (2010), which use the STARBURST99 code, but these grids comprise an insufficiently hard ionizing radiation field, leading to deficiencies in the [S II] fluxes produced by the models.

In galaxy surveys there are at least three methods commonly used to deal with AGNs. The first one consists of removing galaxies hosting AGNs by cross-correlating the sample with published AGN catalogs (e.g. Condon et al. 2002; Serjeant et al. 2002). The second one deals with the identification of the galaxies through the so-called BPT diagram. Nevertheless, at $z \gtrsim 0.5$, the $\mathrm{H} \alpha$ line is redshifted out of the optical range. The third method consists of subtracting AGNs in a statistical manner, used when no other methods are applicable (e.g. Tresse \& Maddox 1998).

In addition to those methods, we propose the use of the S2N2 diagram, which has demonstrated the ability of accurately segregate $\mathrm{SF}$ from composite and AGN galaxies. The S2N2 diagram has the following advantages: an extinction correction is not necessary, since all the emission lines are close in wavelength. It requires only a narrow spectral range, making it suitable for surveys of limited spectral coverage. The SF and composite divisions of the S2N2 diagram offer less contamination in all cases, with respectively a higher number of galaxies for SF and composites than using only the $\mathrm{N} 2$ ratio. Additionally, the user can choose any of the SF galaxy divisions provided for the S2N2 diagram, either when the low contamination from composite galaxies (Eq. (1)) or when selection of the most SF galaxies (Eq. (2)) is required. Finally, the use of the $[\mathrm{S} \mathrm{II}]$ lines does not reduce the number of galaxies, since those lines for AGN galaxies are $~ 1.3$ times stronger than the [O III] line used in the BPT diagrams. Then, comparing the number of galaxies of all the types, the S2N2 diagram has $1 \%$ more galaxies than the $\log ([\mathrm{N} \mathrm{II}] / \mathrm{H} \alpha)$ vs. $\log ([\mathrm{O} \mathrm{III}] / \mathrm{H} \beta)$ diagram. Although this diagram has been used in the past for galaxies, it is the first time that it is presented as a diagnostic diagram for classifying galaxies. Given its advantages, we propose it as an alternative to the BPT diagrams and N2 ratio for classifying galaxies.

\section{Evolutionary effects on the BPT diagrams}

As explained in Sect. 3, the BPT and other optical emission lines diagnostic diagrams have become important in the classification of galaxies. In this section, our aim is to investigate the effects of the evolution of galaxies from the three BPT diagrams. For this purpose, and with the objective of increasing our number of galaxies, we did not allow any restriction in magnitude, as detailed in the sample selection.

In Fig. 5 we show the three BPT diagrams for the four redshift samples. As redshift increases, we observe that [O III] $\lambda 5007 / \mathrm{H} \beta$ goes toward higher values. To explain this shift, we plotted the ratio [O III] $\lambda 5007 / \mathrm{H} \beta$ versus redshift and metallicity in Fig. 6 only for SF galaxies selected with the Kauf03 criterion. The gap observed around $z \sim 0.145$ (see Figs. 6a,c) comes from the $\mathrm{H} \beta$ line falling nearby the $5577 \AA$ sky line, because the residuals are significant and, as a consequence, measurements of $\mathrm{H} \beta$ around this redshift were lost. As shown in Fig. 6b, there is a clear tendency of the [O III] $\lambda 5007 / \mathrm{H} \beta$ ratio towards higher values with redshift, which is explained by examining the same ratio against $12+\log (\mathrm{O} / \mathrm{H})$. The ratio $[\mathrm{O}$ III] $\lambda 5007 / \mathrm{H} \beta$ has been demonstrated to correlate linearly with metallicity (see, for example, Liang et al. 2006). Then, a decrement in $12+\log (\mathrm{O} / \mathrm{H})$ will result in higher values of [O III] $\lambda 5007 / \mathrm{H} \beta$ (see Fig. 6b). We observe a decrement of $\sim 0.2 \mathrm{dex}$ in [O III] $\lambda 5007 / \mathrm{H} \beta$ and a decrement of $\sim 0.1$ dex in $12+\log (\mathrm{O} / \mathrm{H})$ for the $z_{3}$ redshift range with respect to the $z_{0}$ range.

In previous papers (Lara-López et al. 2009a,b), we reported a decrement in $12+\log (\mathrm{O} / \mathrm{H})$ of $\sim 0.1$ dex for the redshift range $0.3<z<0.4$ when comparing galaxies in the same range of luminosity at different redshift intervals. Since the possible bias, such as the luminosity, mass, and aperture effects of those samples, was carefully studied, we demonstrated there that this decrement in metallicity stems from an intrinsic evolution of the galaxies.

Although our $z_{3}$ sample corresponds to luminous galaxies, if we compare galaxies with the same luminosity taking our previous papers as a reference, the metallicity decrement will again be of $\sim 0.1 \mathrm{dex}$, and as consequence, the effects on the BPT diagrams will be the same. Therefore, the evolution observed in the [O III] $\lambda 5007 / \mathrm{H} \beta$ lines ratio toward higher values in the three BPT diagrams could be attributed to evolution in metallicity.

On the other hand, we analyzed the $[\mathrm{N} \mathrm{II}] / \mathrm{H} \alpha$ ratio against redshift and metallicity (see Figs. $6 \mathrm{c}, \mathrm{d}$ ). The $[\mathrm{N} \mathrm{II}] / \mathrm{H} \alpha$ ratio is also a metallicity index, commonly known as N2, and it has been widely studied since it is not severely affected by dust extinction (see Pettini \& Pagel 2004). Among the calibrations of the N2 index, we have for example those of Raimann et al. (2000), Denicoló et al. (2002), and Liang et al. (2006). In Fig. 6d we observe a clear increasing trend of metallicity following the increase in the $\mathrm{N} 2$ index up to $12+\log (\mathrm{O} / \mathrm{H}) \sim 9.0$. The galaxies with $12+\log (\mathrm{O} / \mathrm{H})>9$ show a flattening and a slightly decrease in the N2 index with metallicity (see Fig. 6d). This trend was explained by Kewley et al. (2002) using photoionization models as follows. When the secondary production of nitrogen 
M. A. Lara-López et al.: Evolution from fundamental parameters in SDSS galaxies. II.

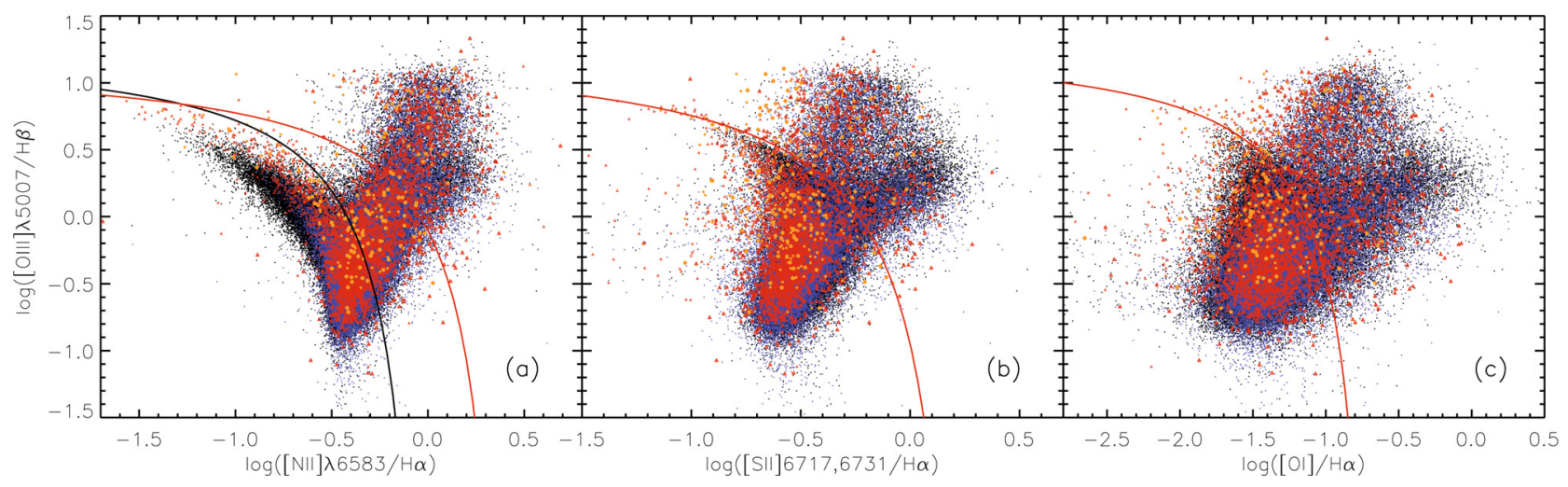

Fig. 5. Evolution of line ratios from the BPT diagrams. Black dots show galaxies in the redshift range $z_{0}$, blue dots galaxies in the $z_{1}$ range, big red triangles represent galaxies in the completeness of the $z_{2}$ range, while small red triangles galaxies out of the completeness, yellow big circles represent galaxies in the completeness of the $z_{3}$ range, and small circles galaxies out of the completeness. The black solid line shows the Kauf03 limit for SF galaxies, and the red solid line shows the Kew01 limit for starburst galaxies in the three BPT diagrams.

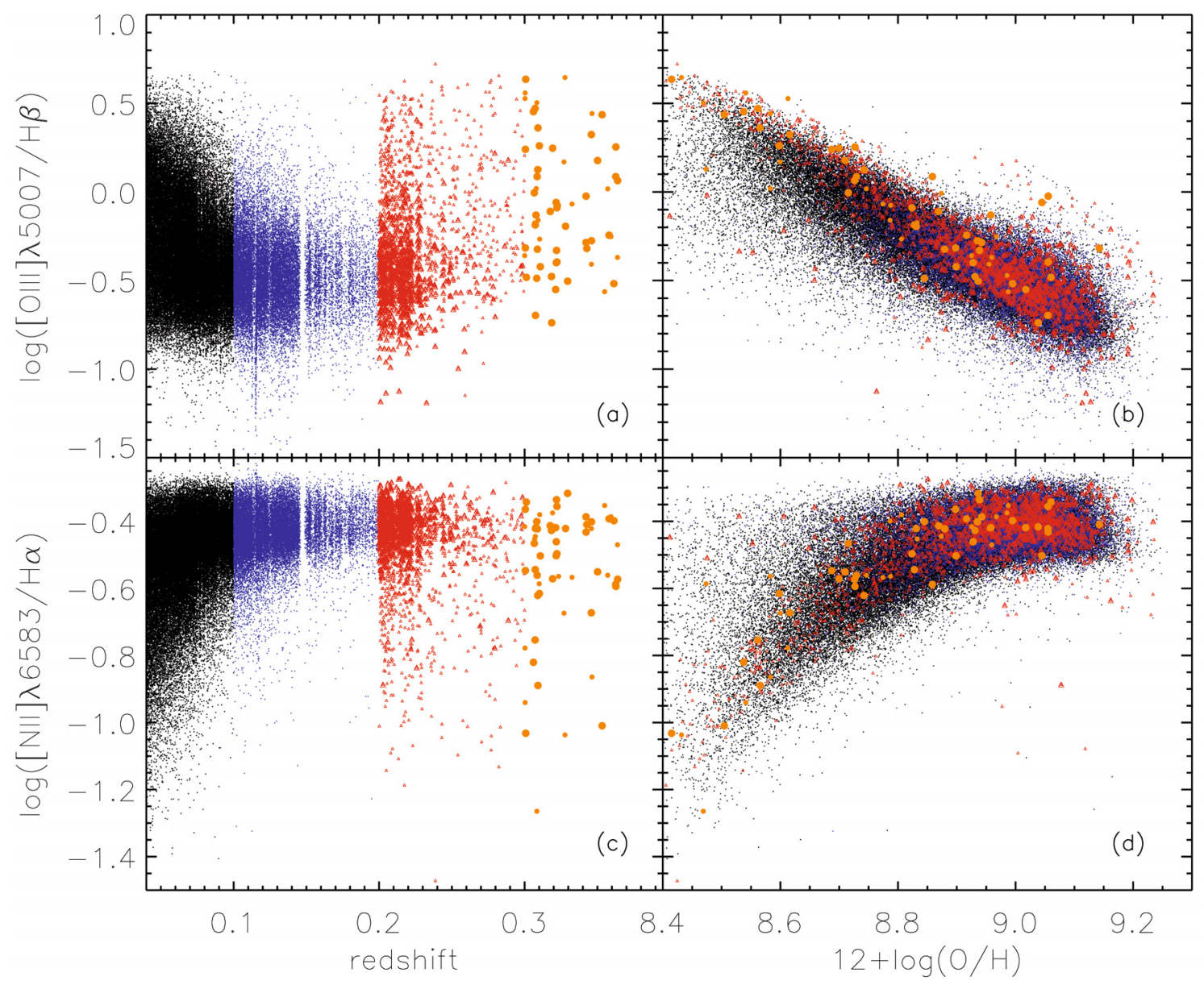

Fig. 6. Evolution with redshift of the $\log ([\mathrm{O} \mathrm{III}] \lambda 5007 / \mathrm{H} \beta)$ ratio for star-forming galaxies (left $)$, and $12+\log (\mathrm{O} / \mathrm{H}) \mathrm{vs}$. $\log ([\mathrm{O} \mathrm{III}] \lambda 5007 / \mathrm{H} \beta)$ for the same galaxies (right). Symbols are the same as in Fig. 5. The gap observed at $z \sim 0.145$ comes from the $5577 \AA$ sky line falling in the $\mathrm{H} \beta$ line, missing galaxies around this redshift.

dominates at somewhat higher metallicity, the $[\mathrm{N}$ II $] / \mathrm{H} \alpha$ line ratio continues to increase, despite the decreasing electron temperature. Eventually, at still higher metallicities, nitrogen becomes the dominant coolant in the nebula, and the electron temperature falls enough to ensure that the nitrogen line weakens with increasing metallicity. Using SDSS galaxies with redshifts $0.04<z<0.25$, Liang et al. (2006) observed a small decrement of the N2 index against metallicity; this turnover of the
$\mathrm{N} 2$ index is more evident for the higher redshifts $z_{2}$ and $z_{3}$ in our sample (see Fig. 6d). The turnover of the $\mathrm{N} 2$ ratio will produce, in the $[\mathrm{O}$ III $] \lambda 5007 / \mathrm{H} \beta$ vs. [N II] $/ \mathrm{H} \alpha$ BPT diagram, the turnover zone around $\mathrm{N} 2 \sim-0.4$, which is more evident in a density plot (see Fig. 2).

Regarding the two left hand BPT diagrams in Figs. 5b and c, since they share the ratio [O III] $\lambda 5007 / \mathrm{H} \beta$, the evolutionary effects due to a decrement in metallicity will be the same as 
discussed above. The ratio $[\mathrm{S} \mathrm{II}] / \mathrm{H} \alpha$ has never been used before as a metallicity indicator because it is far more sensitive to ionization than to metallicity (Liang et al. 2006). Moreover, it is double-valued with metallicity (see Fig. 4), whereas the ratio $[\mathrm{OI}] / \mathrm{H} \alpha$ is not a metallicity indicator.

Therefore, after analyzing all the ratios involved in the three BPT diagrams, we concluded that the evolution of galaxies in the three BPT diagrams is shown through the $[\mathrm{O}$ III $] \lambda 5007 / \mathrm{H} \beta$ ratio. Since this ratio is a metallicity indicator, any decrement in metallicity will result in higher values of the $[\mathrm{O}$ III] $\lambda 5007 / \mathrm{H} \beta$ ratio.

\section{Evolution of the mass-metallicity and luminosity-metallicity relations}

It has been demonstrated that the metallicity and mass of SF galaxies are strongly correlated, with massive and luminous galaxies showing higher metallicities than less massive galaxies (see Sect. 1). The masses of our galaxies were estimated using the STARLIGHT code, which fit an observed spectrum with a combination of 150 SSPs from the evolutionary synthesis models of Bruzual \& Charlot (2003), computed using a Chabrier (2003) initial mass function between 0.1 and $100 M_{\odot}$, and "Padova 1994" evolutionary tracks. The 150 base elements span 25 ages between $1 \mathrm{Myr}$ and $18 \mathrm{Gyr}$, and six metallicities from $Z=0.005$ to $2.5 Z_{\odot}$. As argued by Mateus et al. (2006), including very low $Z$ SSPs in the base inevitably leads to higher stellar masses. A comparison with the Kauffmann et al. (2003b) mass estimates, which are based on a library of model galaxies constructed with $Z>0.25 Z_{\odot}$, results in systematic discrepancies of about 0.1 dex (for details see Mateus et al. 2006). The masses of our galaxies were corrected for aperture effects based on the differences between the total galaxy magnitude in the $r$ band, and the magnitude inside the fiber, assuming that the mass-tolight ratio does not depend on the radius (see Mateus et al. 2006, for details).

A histogram of our mass estimates is shown in Fig. 7, where a larger fraction of massive galaxies are observed at highest redshifts. In Fig. 8 we derived the $M-Z$ and $L-Z$ relations for the galaxies of our sample. As explained in previous sections, galaxies of the $z_{0}$ and $z_{1}$ samples are complete in luminosity, while for the $z_{2}$ and $z_{3}$ samples, the completeness criterium is not taken into account.

The $M-Z$ relation of T04, which is valid over the range $8.5<$ $\log \left(M_{\text {star }} / M_{\odot}\right)<11.5$, shows a steep $M-Z$ relation for masses from $10^{8.5}$ to $10^{10.5} M_{\odot}$ that flattens at higher masses. In this study, T04 analyzed galaxies with redshift ranges $0.005<z<$ 0.3 . It is important to notice that in our $M-Z$ relation, a flatness is not observed for masses $\gtrsim 10^{10.5}$ for the redshift range $z_{0}$, (see Fig. 8a), but this flatness is observed for the higher redshift samples. Then, the flatness observed by T04 depends on the redshift range observed. To establish the bias-free $M-Z$ relation for local galaxies, Kewley \& Ellison (2008) recalibrated the $M-Z$ relation of T04 with galaxies at $0.04<z<0.1$, since 0.04 is the minimum redshift to avoid fibers effects (Kewley et al. 2005).

In Fig. 8 ( $M-Z$ and $L-Z$ relations) the metallicity decrement for the $z_{3}$ redshift sample discussed in our previous articles (Lara-López et al. 2009a,b) is also evident. To fit our local $M-Z$ relation, we estimated the mode of the metallicity of the galaxies in mass bins of $0.1 \mathrm{dex}$ and fit them with a second-order polynomial $\left(y=a_{0}+a_{1} x+a_{2} x^{2}\right)$, with $a_{0}=-0.467, a_{1}=1.611$, $a_{2}=-0.067$. We also fit a second-order polynomial to our $M-Z$ relation for galaxies at $z_{3}$, with $a_{0}=-0.632, a_{1}=1.557$,

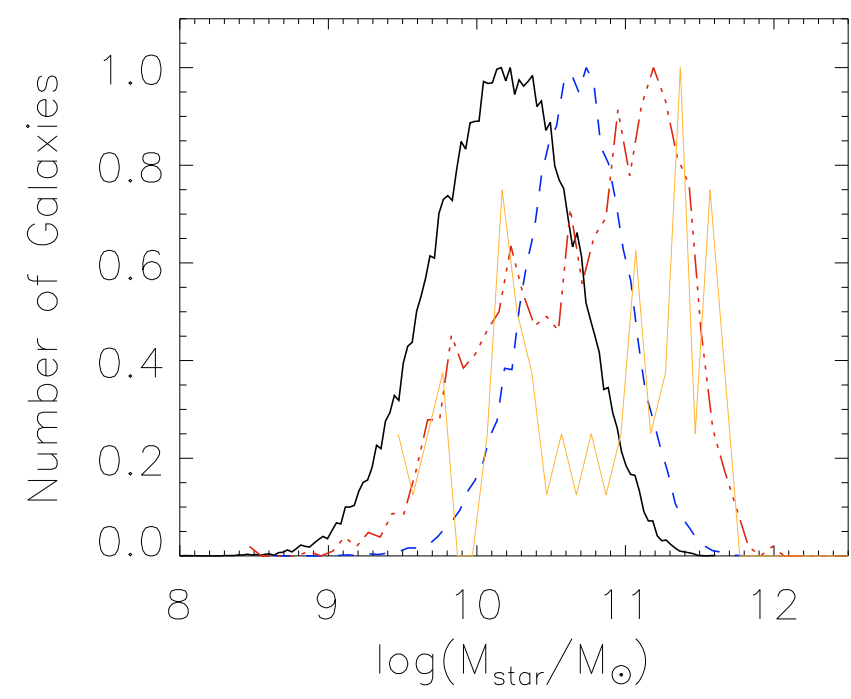

Fig. 7. Normalized mass histogram for all our samples. Dark solid line represent galaxies at $z_{0}$, dashed line galaxies at $z_{1}$, point dashed line galaxies at $z_{3}$, and clear solid line galaxies at $z_{3}$. The histograms were constructed not taking the completeness for galaxies at $z_{2}$ and $z_{3}$ into account. (See the electronic edition of the Journal for a color version of this figure.)

$a_{2}=-0.063$. All the fits are shown in Fig. 9. According to them, our $M-Z$ relation for the galaxies at $z_{3}$ is $\sim 0.2$ dex lower than our local galaxy sample. Additionally, in Fig. 9 we compare the $M-Z$ fits from literature at different redshifts with our results. At $z \sim 0.15$ we represent the calibration of T04, at $z \sim 0.07$ the T04 recalibration of Kewley \& Ellison (2008), as well as our fit for the local $\left(z_{0}\right) M-Z$ relation. At higher redshift, we represent our fit to the $M-Z$ relation for galaxies at $z_{3}$, as well as the fit of Erb et al. (2006a) at $z \sim 2.2$ scaled to the T04 metallicity calibration. Due to their high redshift, Erb et al. (2006a) used the N2 method and the calibration of Pettini \& Pagel (2004) to estimate their metallicities. We converted their N2 metallicities to the $R_{23}$ calibration of T04 with the metallicity conversions given in Kewley \& Ellison (2008). Even with the dispersion of our local sample, our $M-Z$ fit is a little lower, but in a good agreement with those of T04 and Kewley \& Ellison (2008). Since we are using the T04 calibration of the $\mathrm{R}_{23}$ method to estimate metallicities, the main differences with the fit of T04 are the redshift ranges, as discussed above, and the mass estimates, since T04 and Kewley \& Ellison (2008) adopted a Kroupa et al. (2001) IMF, while we are using a Chabrier (2003) IMF.

Although redshift ranges are different, the comparison of our fit for the $z_{3}$ sample with the Erb et al. (2006a) data at $z \sim 2.2$, which also use a Chabrier IMF, are similar in $12+\log (\mathrm{O} / \mathrm{H})$ (see Fig. 9). As will be explained in the next sections, our $z_{3}$ sample is composed mainly of spiral galaxies, while the Erb et al. (2006a) sample corresponds to a mix of morphological types. A possible explanation for the high metallicities of Erb et al. (2006a) or to the lower metallicities of our sample is given by Calura et al. (2009), who use models that distinguish among different morphological types through the use of different infall, outflow, and star formation to reproduce the mass-metallicity relation in galaxies of all morphological types, taking the observational $M-Z$ relations of Kewley \& Ellison (2008), Savaglio et al. (2005), Erb et al. (2006a), and Maiolino et al. (2008) as a reference. In his work Calura et al. (2009) predicts that, at any redshift, elliptical galaxies will present the highest stellar masses and the highest metallicities, whereas the irregulars are the least 


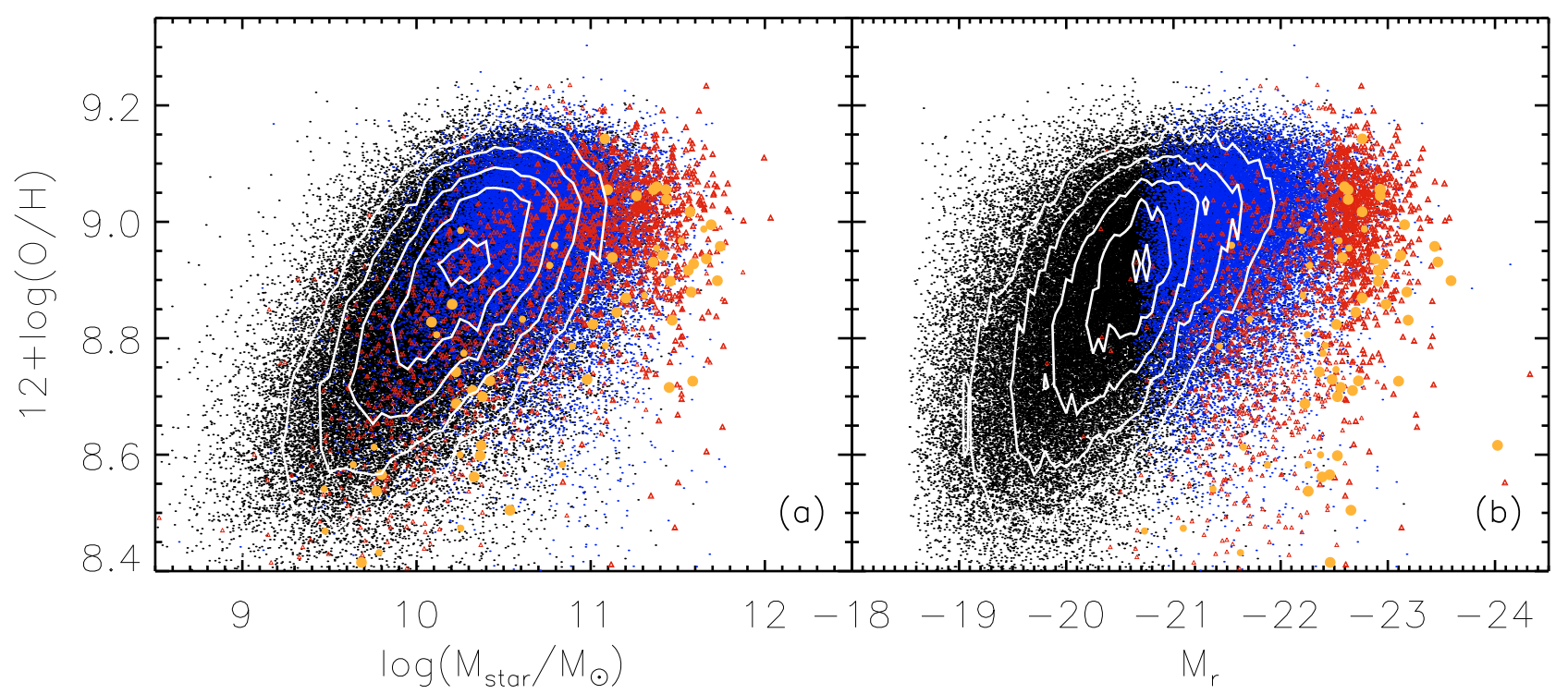

Fig. 8. a) Relation between the stellar mass and $12+\log (\mathrm{O} / \mathrm{H})(M-Z$ relation). b) Relation between the absolute Petrosian $r$ magnitude and $12+\log (\mathrm{O} / \mathrm{H})\left(L-Z\right.$ relation) for our sample of galaxies. The cut observed in Fig. b) for the $z_{1}$ sample comes from the $5577 \AA$ sky line (see the text). In both relations, white contours represent from outside to inside, $15,30,50,70$, and $90 \%$ of the maximun density value of the $z_{0}$ redshift sample (black dots) and are only plotted as a visual aid. Colors and symbols follow the same code used in Fig. 5. (See the electronic edition of the Journal for a color version of this figure.)

massive and metallic galaxies, with spiral galaxies being at an intermediate stage.

This means that, because our $z_{3}$ sample is composed only of spiral galaxies, our metallicities will be lower than if our sample were composed of a mix of morphological types. The observed metallicities and SFRs for the Erb et al. (2006a) sample at $z \sim 2.2$, according to the study of Calura et al. (2009), indicate that their galaxies are likely to represent a morphological mix, partly composed of spirals (or proto-spirals) and partly of ellipticals (or proto-ellipticals). Calura et al. (2009) predicts lower metallicities $(\sim 0.3 \mathrm{dex})$ for the Erb et al. (2006a) galaxies at $z \sim 2.2$ if the sample were composed of spiral galaxies. Then, the similarities in $12+\log (\mathrm{O} / \mathrm{H})$ of our $M-Z$ relation at $z_{3}$ with the one of Erb et al. (2006a) at $z \sim 2.2$, could be explained by the morphological selection in each case: lower metallicities in our $M-Z$ relation can be addressed to the prominence of spiral galaxies, whereas higher metallicities in the $M-Z$ relation of the Erb et al. (2006a) is a consequence of a sample formed by a mix of morphological types. In other words, the morphology of the galaxies is crucial in deriving and comparing the metallicity and the $M-Z$ relation.

An additional point is that our samples plotted in Fig. 9 are selected with different magnitude completeness, then, our $z_{3}$ redshift sample is more luminous and massive than our local one. This must be taken into account when comparing both $M-Z$ relations. Unfortunately, our $z_{0}$ sample does not have enough galaxies in the same absolute magnitude range of the $z_{3}$ sample, making it imposible to generate a local $M-Z$ relation comparable in luminosity to the $z_{3}$ one.

Comparing our $M-Z$ relation with that of high- $z$ samples in the literature, we have the $M-Z$ relation of Savaglio et al. (2005) at $z \sim 0.7$. In their study, they only find a slight decrement in metallicity for galaxies at $z \sim 0.7$ compared with the local one of T04, which is inconsistent with the $\sim 0.2$ dex decrement found in our $z_{3} M-Z$ relation. Nevetheless, as pointed out by Rodrigues et al. (2008), the sample of Savaglio et al. (2005) have spectra with a low $\mathrm{S} / \mathrm{N}$ and spectral resolution, as well as extinction problems. With a more consistent result, Rodrigues et al. (2008) generated the $M-Z$ relation for galaxies at $z \sim 0.7$, finding a decrement in metallicity of $\sim 0.3$ dex compared with the local one of T04. Unfortunately, given the narrow range in their stellar masses, it was impossible to constrain the evolution of the shape of their $M-Z$ relation.

There are two main ways to explain the origin of the $M-Z$ relation. The first one is related to the well-known effect of downsizing (e.g. Cowie et al. 1996; Gavazzi \& Scodeggio 1996), in which lower mass galaxies form their stars later and on longer time scales than more massive systems, implying low star formation efficiencies in low-mass galaxies (Efstathiou 2000; Brooks et al. 2007; Mouhcine et al. 2008; Tassis et al. 2008; Scannapieco et al. 2008; Ellison et al. 2008). Therefore, low-mass galaxies are expected to show lower metallicities. Supporting this scenario, Calura et al. (2009) have reproduced the $M-Z$ relation with chemical evolution models for ellipticals, spirals, and irregular galaxies by means of an increasing efficiency of star formation with mass in galaxies of all morphological types, without the need for outflows favoring the loss of metals in the less massive galaxies. In a recent study that supports this result for massive galaxies, Vale Asari et al. (2009) model the time evolution of stellar metallicity using a closed-box chemical evolution picture. They suggest that the $M-Z$ relation for galaxies in the mass range from $10^{9.8}$ to $10^{11.65} M_{\odot}$ is mainly driven by the star formation history and not by inflows or outflows.

A second scenario to explain the $M-Z$ relation is attributed to metal and baryon loss from gas outflow, where low-mass galaxies eject large amounts of metal-enriched gas by supernovae winds before high metallicities are reached, while massive galaxies have deeper gravitational potentials that help to retain their gas, thus reaching higher metallicities (Larson 1974; Dekel \& Silk 1986; MacLow \& Ferrara 1999; Maier et al. 2004; T04; De Lucia et al. 2004; Kobayashi et al. 2007; Finlator \& Dave 2008). As pointed out in the high-resolution simulations of Brooks et al. (2007), supernovae feedback plays a crucial role in lowering the star formation efficiency in low-mass galaxies. 


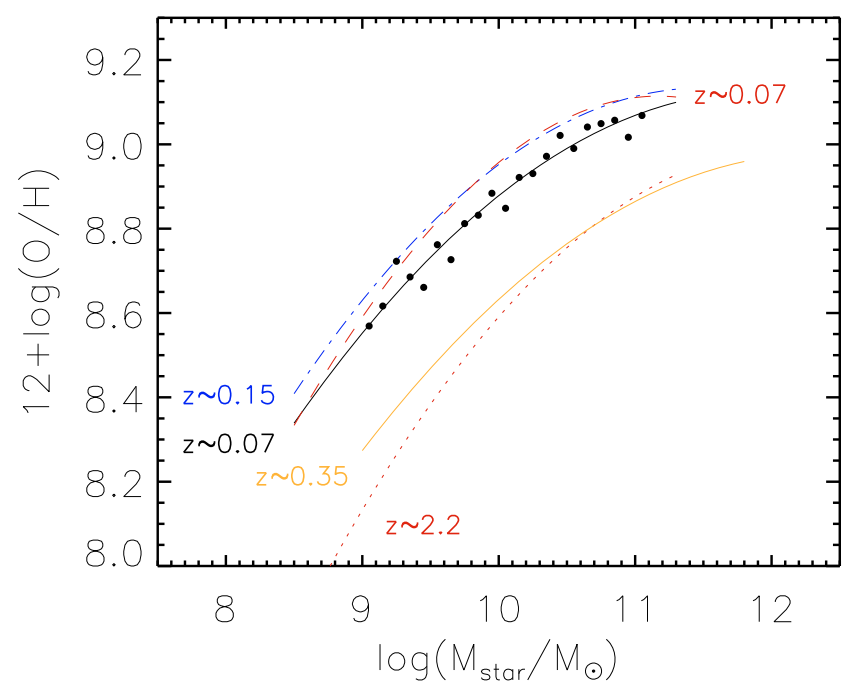

Fig. 9. Evolution of the mass-metallicity relation observed at different redshifts. The point-dashed line represents the curve of T04 at $z \sim 0.15$, the dashed line the T04 recalibration of Kewley \& Ellison (2008) at $z \sim 0.07$, circles are the mode metallicity in $\log \left(M_{\text {star }} / M_{\odot}\right)$ bins of 0.1 for our $z_{0}$ sample, the solid dark and clear curves represent our fit for $z_{0}$ (mode bins) and $z_{3}$, respectively. The dotted line represents the fit of Erb et al. (2006a) at $z \sim 2.2$. (See the electronic edition of the Journal for a color version of this figure.)

Without energy injection from supernovae to regulate the star formation, gas that remains in galaxies rapidly cools, forms stars, and increases its metallicity too early, producing a $M-Z$ relation that is too flat compared to observations.

An additional interpretation of the $M-Z$ relation is linked to some properties of star formation, for instance, the IMF. Köppen et al. (2007) suggest that the $M-Z$ relation can be explained by a higher upper-mass cutoff in the IMF in more massive galaxies.

Finally, we also generated the $L-Z$ relation for our redshift samples (see Fig. 8b). Nevertheless, our $z_{3}$ sample is restricted to a narrow range in luminosity, making it impossible to fit a curve. The local $L-Z$ relation is well established by T04, then, due to our small luminosity range at $z_{3}$ we cannot conclude anything about the $L-Z$ relation. Nevertheless, the $M-Z$ relation has shown itself to be stronger and tighter than the $L-Z$ relation, confirming that stellar mass is a more meaningful physical parameter than luminosity when both are compared with gas metallicity (Savaglio et al. 2005).

\subsection{Evolution of the mass-to-light ratio}

As explained by Erb et al. (2006a), at higher redshifts, the $M-Z$ relation is clearly more physically meaningful than the $L-Z$ relation. A corollary is that the local $L-Z$ relation is simply the result of the strong correlation between mass and luminosity at low redshift.

We also analyzed the evolution of the mass-to-light $(M / L)$ ratio (see Fig. 10). For a given mass, we observed higher luminosities for the $z_{3}$ sample compared with the local one, which means lower $M / L$ ratios as redshift increases. To observe this evolution, we fit a line $\left(y=a_{0}+a_{1} x\right)$ to the $z_{0}$ and $z_{3}$ redshift samples (see Fig. 10), obtaining $a_{0}=-0.924$ and $a_{1}=-0.544$ for $z_{0}$, and $a_{0}=-18.686$ and $a_{1}=-1.304$ for $z_{3}$. The variation in $M / L$ at a given rest frame optical luminosity can be as much as a factor of $\sim 70$ (Shapley et al. 2005), which means that an extended range of stellar masses exists for any range in

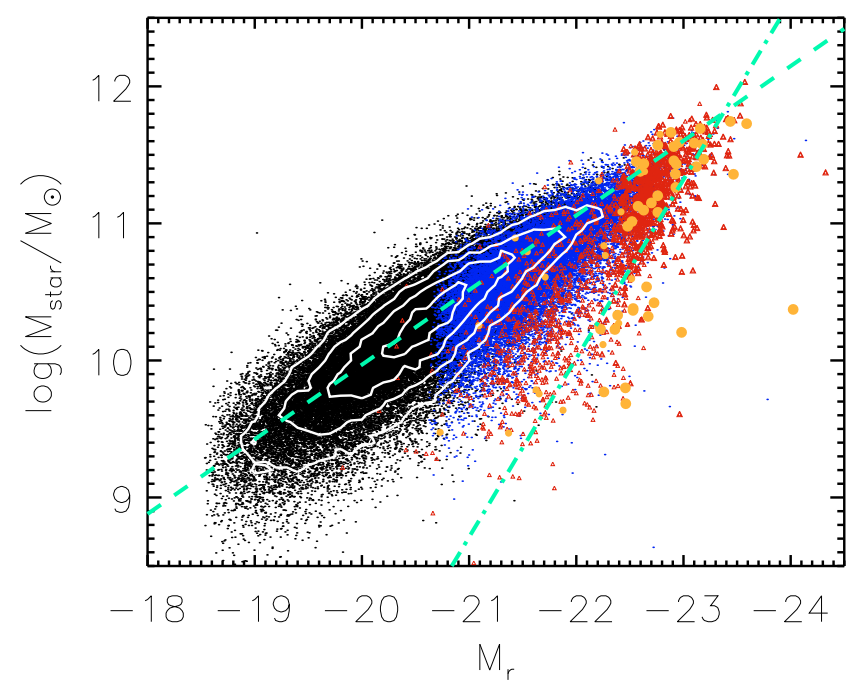

Fig. 10. Mass versus Petrosian absolute $k$-corrected magnitude for all our redshift samples. White contours represent, going from outside to inside, $10,25,50$, and $80 \%$ of the maximun density value of the $z_{0}$ sample. The dashed line represents the fit to the $z_{0}$ sample, while the pointdashed line is the fit to the $z_{3}$ sample. Colors and symbols follow the same code as in Fig. 5. (See the electronic edition of the Journal for a color version of this figure.)

luminosity. This large variation in $M / L$ explains the lack of correlation in the $L-Z$ relation for the $z_{3}$ sample compared to the local relation. For a narrow range of absolute magnitudes in the $z_{3}$ sample, we have a wide range of masses, making it possible to generate an $M-Z$ relation. At higher redshifts, the effect is the same, as pointed out by Erb et al. (2006a), finding a wide range of stellar mass in a narrow range of luminosity for star-forming galaxies at $z \sim 2.2$.

\section{Morphology indicators and SFR}

The variation in SFR activity and young stellar content along the Hubble sequence is one of the most recognizable features of galaxies. In fact, this variation in stellar content is part of the basis of the Hubble classification itself (Hubble 1926), and understanding its physical nature and origin is fundamental to comprehending the galaxy evolution (for reviews see Kennicutt 1998; Kennicutt et al. 1994). The general picture, presented by Roberts (1963), Searle et al. (1973), Larson \& Tinsley (1978), and Kennicutt et al. (1994), shows that early-type galaxies (types S0$\mathrm{Sb}$ ) represent systems that formed most of their gas into stars on timescales much less than the Hubble time, while the disks of late-type systems $(\mathrm{Sc}-\mathrm{Im})$ have formed stars at a roughly constant rate since they formed.

For most of the 20th century, catalogs of morphologically classified galaxies were compiled by individuals or small teams of astronomers (e.g. Sandage 1961; de Vaucouleurs 1991). Nowadays, selection criteria are based on galaxy properties such as color, concentration index, spectral features, surface brightness profile, other structural parameters, or some combination of these (e.g. Strateva et al. 2001; Abraham et al. 2003; Kauffmann et al. 2004; Conselice 2006; Scarlata et al. 2007). With the advent of modern surveys, such as the SDSS, and with the participation of thousands of volunteers, it has been possible to develop the Galaxy Zoo project (e.g. Lintott et al. 2008), providing visual morphological classification for more than $10^{7}$ galaxies. 
M. A. Lara-López et al.: Evolution from fundamental parameters in SDSS galaxies. II.

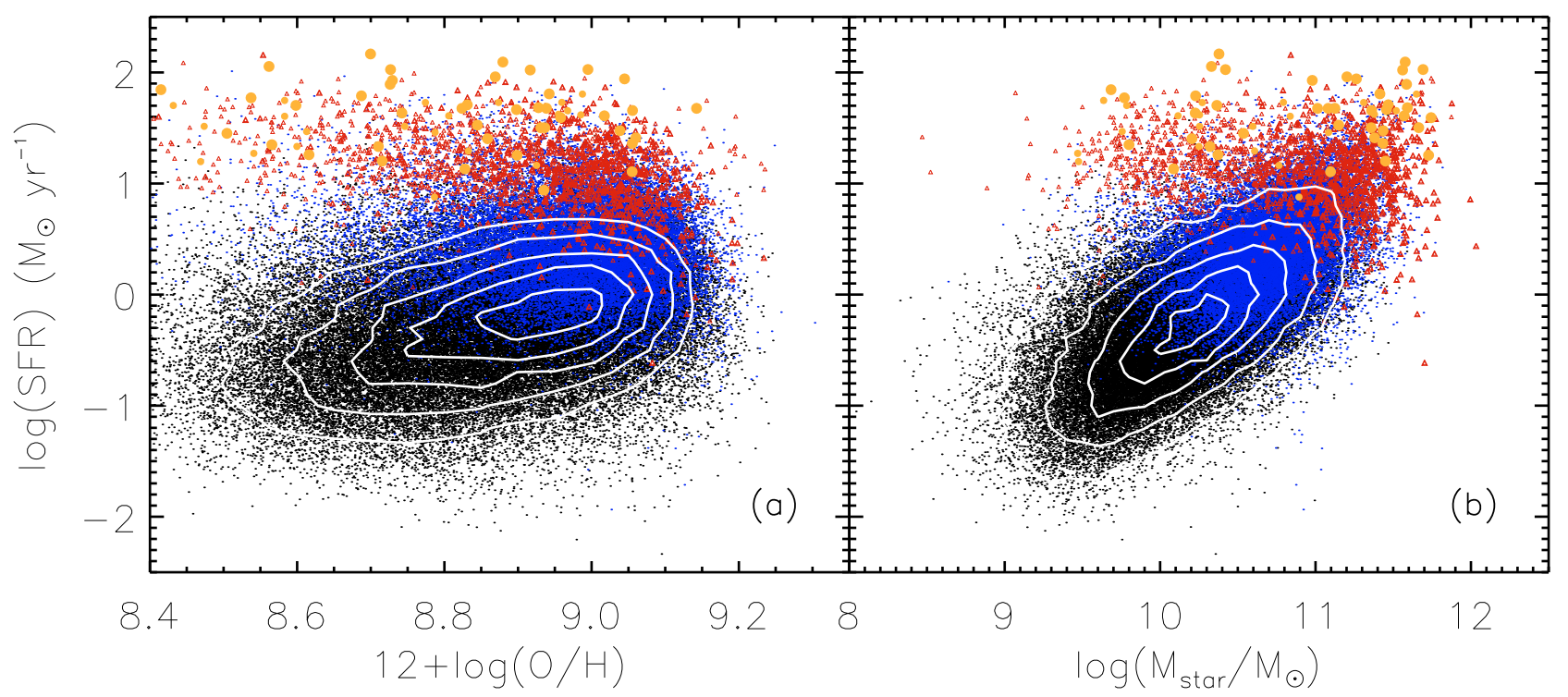

Fig. 11. Metallicity and mass versus $\log (\mathrm{SFR})$. Contours correspond to the $z_{0}$ sample in both plots. White contours represent, from outside to inside, in panel a): $15,30,50,70$, and $90 \%$, and in panel b): $5,15,35,65$, and $85 \%$ of the maximun density value of the $z_{0}$ sample. Colors and symbols follow the same code as in Fig. 5.

In this section, we focus on the relations at different redshifts between the SFR, metallicity, mass, and morphology of SF galaxies selected with the Kauffmann et al. (2003a) criteria, as explained in Sect. 2.

\subsection{Evolution of the SFR}

We estimate the SFR with the $\mathrm{H} \alpha$ emission line flux following the Kennicutt (1998) expression:

$$
\operatorname{SFR}\left[M_{\odot} \mathrm{yr}^{-1}\right]=7.9 \times 10^{-42} L(\mathrm{H} \alpha)\left[\mathrm{erg} \mathrm{s}^{-1}\right],
$$

where $L(\mathrm{H} \alpha)$ denotes the intrinsic $\mathrm{H} \alpha$ luminosity, and $\mathrm{H} \alpha$ is corrected by dust extinction and underlying stellar absorption as explained in Sect. 2. This calibration is derived from evolutionary synthesis models that assume solar metallicity and no dust, and it is valid for a $T_{\mathrm{e}}=10^{4} \mathrm{~K}$ and a case $\mathrm{B}$ recombination

Recent studies have explored the relationship between the stellar mass and the SFR in galaxies at different redshifts. It has been shown that SFR critically depends on the galaxy mass both at low and high redshifts (e.g. Gavazzi et al. 2002; Brinchmann et al. 2004; Dickinson et al. 2004; Feulner et al. 2005; Papovich et al. 2006).

In our sample, galaxies with high SFRs are more abundant at higher redshifts (see Fig. 11), a fact already observed in non biased samples (e.g. Noeske et al. 2007a). In Fig. 11a, we show $12+\log (\mathrm{O} / \mathrm{H})$ against $\log (\mathrm{SFR})$. Although our $z_{3}$ sample of galaxies is biased to the most luminous and massive galaxies, the observed decrement of $\sim 0.1$ dex in $12+\log (\mathrm{O} / \mathrm{H})$ found in Lara-López et al. (2009a,b) is also present. Regarding the $z_{0}$ sample of galaxies, there is a clear sequence with galaxies going toward higher values of SFR as metallicity increases. This tendency can be explained from the $z_{0}$ sample in the $M-Z$ relation of Fig. 8, where massive galaxies correspond to the highest metallicity galaxies, and for more massive galaxies, we expect higher SFRs (see Fig. 11). Also, we can slightly appreciate a population of galaxies with higher SFR (see Fig. 11a). This population will form a tail when the mass is taken into account, as will be shown in Fig. 11b. As redshift increases, we appreciate a flattening of the SFR vs. $12+\log (\mathrm{O} / \mathrm{H})$ relation in Fig. 11a for galaxies at $z_{2}$ and $z_{3}$, with most of the galaxies showing $\log (\mathrm{SFR})$ between 1 and 2 .

In Fig. 11 b, we show the $\log \left(M_{\text {star }} / M_{\odot}\right)$ versus $\log (\mathrm{SFR})$ plot. Galaxies at $z_{0}$ show a main sequence, where massive galaxies have higher SFRs. This main sequence was identified by Noeske et al. (2007a), when studying galaxies with redshifts from 0.2 to 1.1 , finding that this main sequence moves as a whole to higher SFR as redshift increases. The SSFR, defined as the total SFR divided by the stellar mass, reflects the strength of the current burst of star formation relative to the underlying galaxy mass. Deep galaxy surveys have consistently found that the SSFR depends strongly on both $M_{\odot}$ and redshift, with the bulk of star formation occurring earlier in massive galaxies than in less massive systems (Guzmán et al. 1997; Brinchmann \& Ellis 2000; Juneau et al. 2005; Bauer et al. 2005; Bell et al. 2005; Pérez-González et al. 2005; Feulner et al. 2005; Papovich et al. 2006; Caputi et al. 2006; Reddy et al. 2006).

We also analyzed the evolution of the SSFR as a function of the metallicity and the stellar mass. Interestingly, in the $12+\log (\mathrm{O} / \mathrm{H})$ vs. SSFR diagram (Fig. 12a), the observed population of Fig. 11a at $z_{0}$ is more evident, showing a higher SSFR $(S S F R>-10)$ than the other galaxies at the same redshift. We are going to investigate this tail in more detail in the next subsection.

In Fig. $12 \mathrm{~b}$, we show the $\log \left(M_{\text {star }} / M_{\odot}\right)$ versus $\log (\mathrm{SSFR})$ plot. The SSFR increase with redshift, showing for more massive galaxies a tendency toward lower SSFR values, which agrees with the results of Noeske et al. (2007b), for galaxies with $z>0.2$. Massive galaxies shows lower SSFR because they probably have low gas fractions so have nearly finished assembling their stellar mass (Erb et al. 2006b; Reddy et al. 2006). On the other hand, the presence of dust has demonstrated to play an important role when deriving the SSFR, as shown by Pannella et al. (2009), who obtain a flat SSFR for dust free galaxies for redshift bins centered on $z \sim 1.6$ and 2.1, instead of a drop with increasing mass. In our samples, the SFR and SSFR were derived through the dust-corrected $\mathrm{H} \alpha$ flux, showing that this flatness is absent for redshifts below 0.4 . 


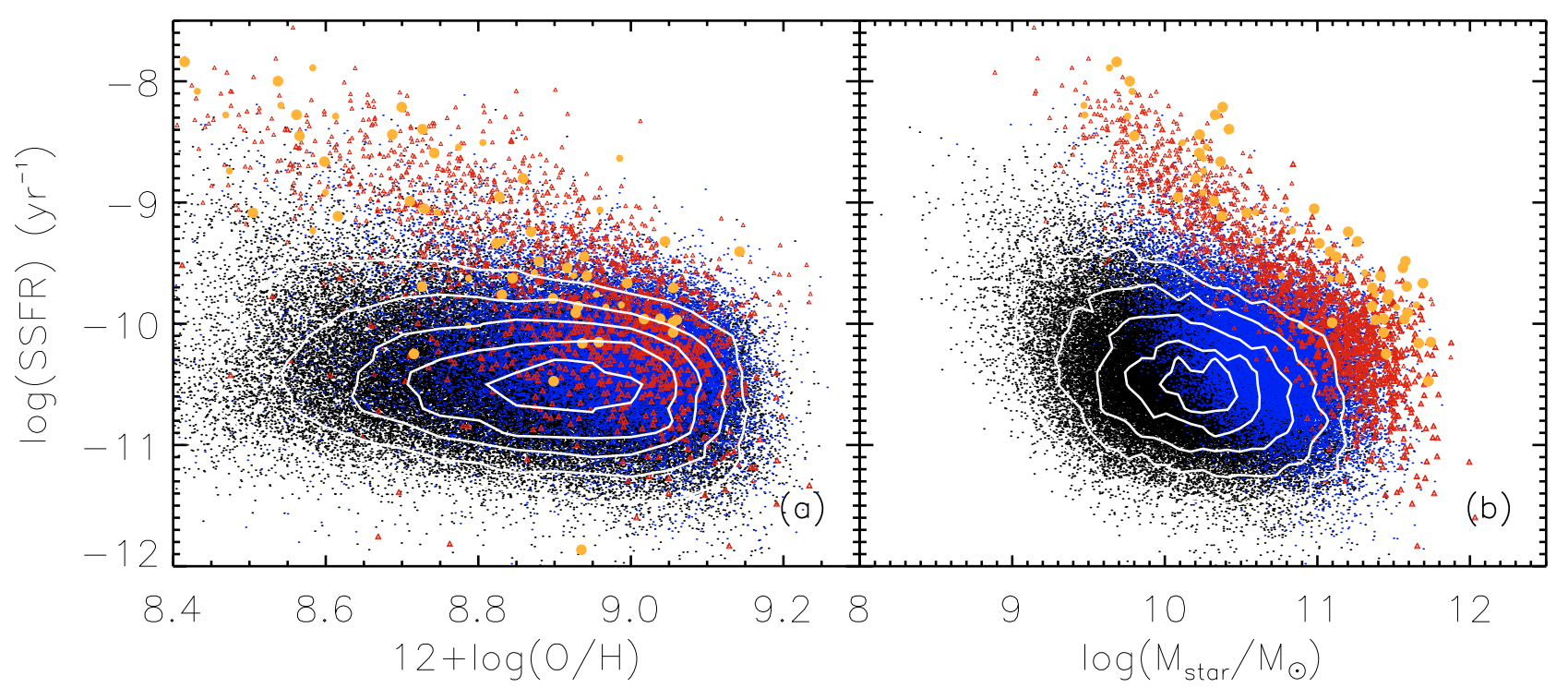

Fig. 12. Metallicity and mass versus $\log (\mathrm{SSFR})$. Contours correspond to the $z_{0}$ sample in both plots. White contours represent, from outside to inside, in panel a): 10, 20, 40,60, and 90\%, and in panel b): 5, 15, 35, 60, and $80 \%$ of the maximun density value of the $z_{0}$ sample. Colors and symbols follow the same code as in Fig. 5.

We found also evidence of two populations in Fig. 12b, one at redshift $z_{0}$, which concentrate in a square delimited by the contour plots, and another one showing $S S F R>-10$, which is more evident for the higher redshifts samples, but is also evident in the $z_{0}$ sample. In a forthcoming paper (Lara-López et al. 2010), we have shown that the SFR, metallicity, and stellar mass of SF galaxies form a fundamental plane (FP) in a 3D space, obtaining a $1 \sigma$ error of 0.16 dex in the mass estimates through the FP. This population of galaxies will be explained in the next section as a consequence of the morphology of the galaxies.

\subsection{The SSFR as a morphology indicator}

In this section, we investigate the morphology of the galaxies in our sample with the aim of clarify whether the tail observed in Figs. 11 and 12, with higher SFR and SSFR in the $z_{0}$ sample, respectively, is related to specific morphological types. We focus on samples $z_{0}$ and $z_{3}$ because those samples show their galaxies uniformly distributed on mass. Galaxies of samples $z_{1}$ and $z_{2}$ show systematic problems due to sky lines and to incompleteness, respectively. We used the $g-r$ color, the concentration index $c=R_{90} / R_{50}$ (e.g. Park \& Choi 2005), and the SSFR (e.g. Salim et al. 2009), which are the most common indexes to segregate early from late-type galaxies (see Fig. 13). We used $\mathrm{k}$-corrected fiber colors for all the galaxies samples.

The color index has been commonly used as an early and late type morphological classifier (Baldry et al. 2004; Faber et al. 2007; Wang et al. 2007; Lee et al. 2007a). Strateva et al. (2001) find that the integrated observed frame $u-r$ shows a bimodal distribution; however, they show that, when divided at $u-r=2.22$, the early and late type subsets have significant contamination, reaching about $30 \%$ for a sample with visually identified morphological types. Because the $u$ band shows large errors for the SDSS galaxies, we decided to use $g-r \lesssim 0.6$ (e.g. Schawinski et al. 2009; Masters et al. 2009), which allows separation of early from late-type galaxies. As observed in Fig. 13a, 97\% of the $z_{3}$ sample of galaxies corresponds to a late-type morphology.

The concentration index $c=R_{90} / R_{50}$ has been successfully used in segregating late $(c<2.86)$ from early-type $(c \geq 2.86)$ subsets (e.g. Shimasaku et al. 2001; Strateva et al. 2001; Goto et al. 2002; Nakamura et al. 2003; Deng et al. 2009). Nevertheless, contamination in the early and late-type subsets separated using the concentration index, is typically about $20 \%$ (Yamauchi et al. 2005; Shimasaku et al. 2001). Using both color and concentration indices, Park \& Choi (2005) used the color-color space $u-r$ versus $\Delta(g-r)$ and the concentration index $c^{-1}=R_{50} / R_{90} \sim 0.35$ as a reliable morphological classifier. In Fig. 13b, we show the concentration index $c$ vs. $g-r$ for galaxies at $z_{0}$ and $z_{3}$, with the $\sim 95 \%$ of the $z_{3}$ galaxies corresponding to late-type $(c<2.86)$ galaxies.

Finally, the SSFR has been used as an indicator of early and late-type morphology (e.g. Wolf et al. 2009; Salim et al. 2009) since late-types have blue colors and high SSFRs, while early-types have red colors and low SSFRs. For our $z_{3}$ sample, $\sim 89 \%$ of the galaxies have $\log (S S F R)>-10$ (see Fig. 13c), and as reported by Salim et al. (2009), blue actively star-forming galaxies have $\log (S S F R)>-10$, while lower values would correspond to the green valley and red-sequence galaxies. As argued by Weinmann et al. (2006), the use of the SSFR would give us important clues for determining the morphological galaxy type, since for example, a genuine SF disk galaxy may appear red owing to strong extinction (e.g. when seen edge-on), and thus be classified as early-type based on its color, while the SFR and morphology quantifiers would classify it as a late-type galaxy.

Our sample of galaxies at $z_{3}$ is mainly composed by late-type galaxies, as indicated by any of the morphological classifiers discussed above. We decided to take these galaxies as a reference to delimit the late-type zone. After trying all the discussed methods, in order to obtain a reliable morphological classification, and following the location of the $z_{3}$ galaxies in Fig. 13c, we conclude that the best method is to use both $\log (S S F R)>-10$ and a color $g-r<0.6$. After applying this criterion to our $z_{0}$ sample, we end with 7967 galaxies classified as late-type, corresponding to $\sim 13 \%$ of the original sample. If we repeat the comparative analysis between SFR and metallicity, and SFR against stellar mass (see Fig. 14) for galaxies at $z_{0}$ and $z_{1}$, the tail with higher SFR identified in previous sections corresponds to latetype galaxies. The separation between late and early-type in the 
M. A. Lara-López et al.: Evolution from fundamental parameters in SDSS galaxies. II.

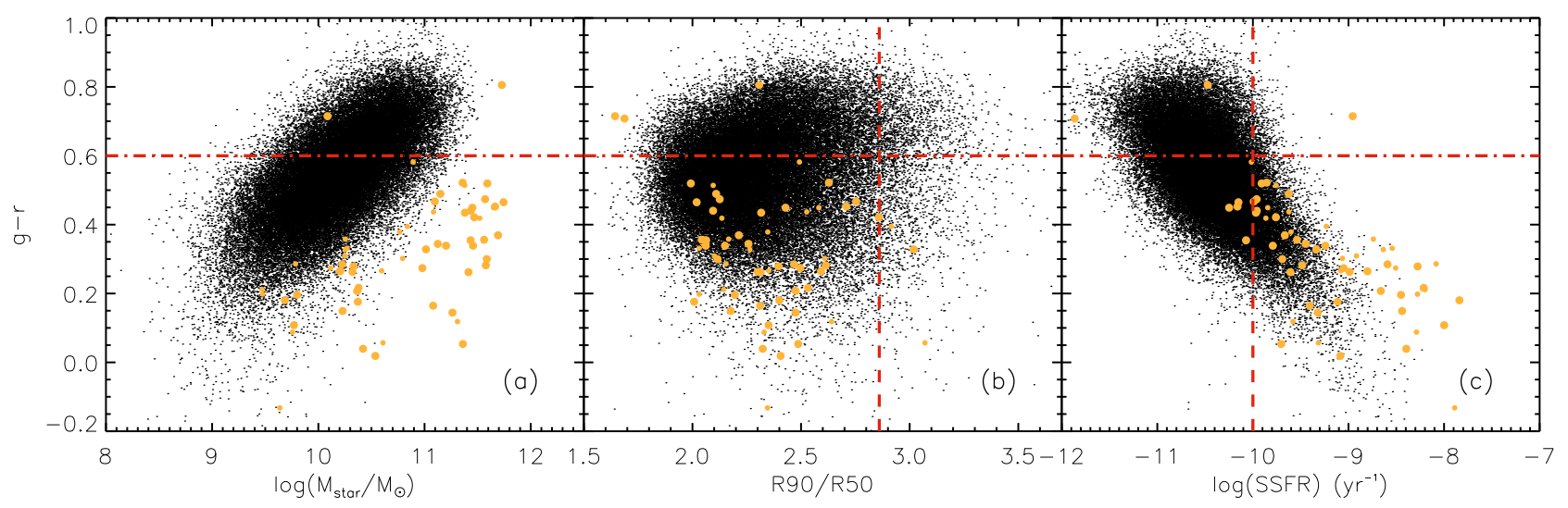

Fig. 13. Principal morphological classifiers. In panels a)-c), we show the $\log \left(M_{\text {star }} / M_{\odot}\right)$, concentration index $c=R_{90} / R_{50}$, and $\log (\mathrm{SSFR})$ versus $g-r$ color, respectively. Black dots and yellow circles represent galaxies at $z_{0}$ and $z_{3}$, respectively. In each panel the dashed line shows the standard limit for segregating early from late-type galaxies, with the $z_{3}$ galaxies concentrated in the late-type region in each panel. (See the electronic edition of the Journal for a color version of this figure.)

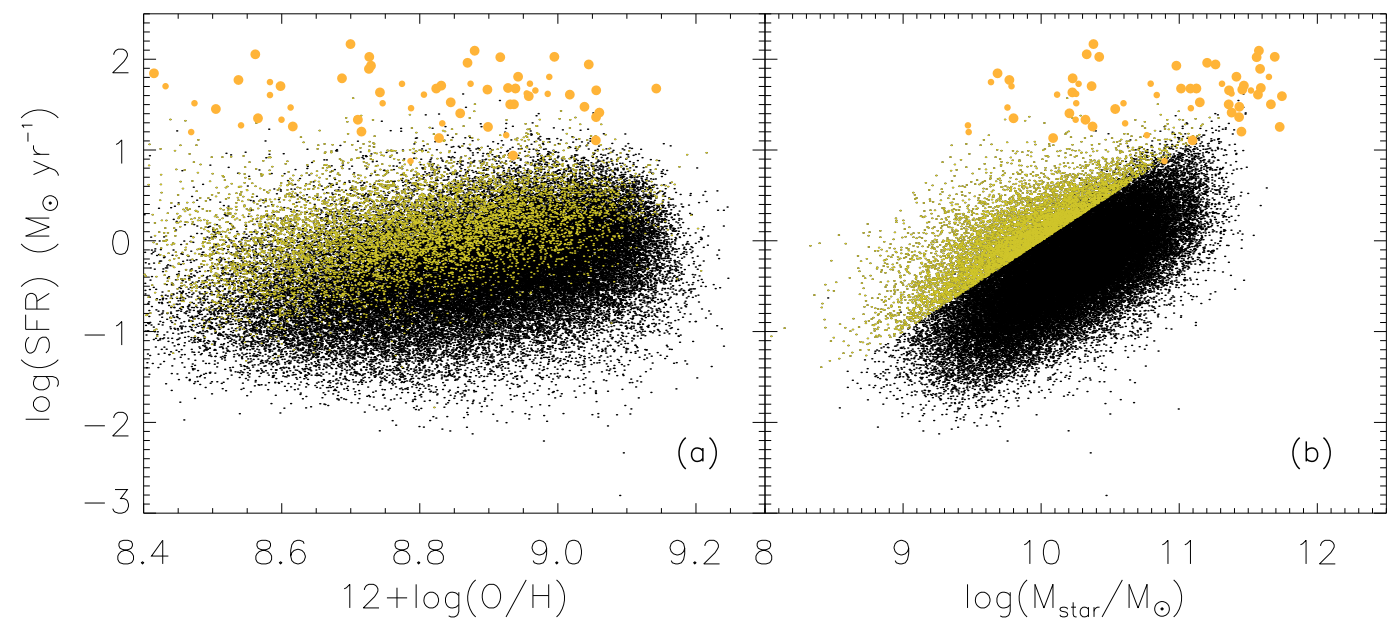

Fig. 14. Metallicity and mass versus $\log (\mathrm{SSFR})$. Black dots and yellow circles represent galaxies at $z_{0}$ and $z_{3}$, respectively, while green dots, represent late-type galaxies at $z_{0}$ selected with $\log (S S F R)>-10$. (See the electronic edition of the Journal for a color version of this figure.)

stellar mass versus SFR is a straight border (see Fig. 14b), because the separation criterion is the SSFR.

To alternatively assess this statement, we compared our results with selected mock galaxy samples from the Millennium simulations (Springel et al. 2005). We used the Bower2006a catalog (Bower et al. 2006), which gives us redshift, SDSS $k$-corrected colors, stellar mass, and $\mathrm{H} \alpha$ luminosity, among other information. From the original catalog, we randomly selected 35000 galaxies at $z=0$ and $z=0.4$ with the $\mathrm{H} \alpha$ line in emission to be able to compare with our galaxies. As shown in Fig. 15, there is a clean separation between a red sequence, formed by galaxies with $g-r \gtrsim 0.8$, the green valley with $0.6 \lessgtr g-r \lessgtr 0.8$, and the blue cloud with $g-r \lessgtr 0$.6. It can be observed how galaxies move towards late-type galaxies as redshift increases. Then, these results are consistent with mainly observing latetype galaxies at redshift $z_{3}$.

\section{Summary and conclusions}

We analyzed a sample of emission line galaxies selected in four redshift intervals from $\sim 0$ to 0.4 in bins of 0.1 , taking the magnitude completeness of every redshift interval into account. In this paper we introduced the S2N2 diagram as a star-forming, composite, and AGN galaxy classifier, estimated metallicities using the $R_{23}$ method, and analyzed the evolutive effects of galaxies from the three BPT diagrams. Additionally, we studied the evolution of the $M-Z$ and $L-Z$ relations and analyzed the evolution and implications of the galaxy morphology in the SFR-mass and metallicity relations. From these analyses we conclude the following.

- Using the Kew01 photoionization grids, the Kauf03 and Kew01 SF, and starburst limit respectively, in the $[\mathrm{N} \mathrm{II}] / \mathrm{H} \alpha$ vs. [O III] $\lambda 5007 / \mathrm{H} \beta$ diagram, we demonstrated that the $\mathrm{S} 2 \mathrm{~N} 2$ is a well-behaved diagnostic diagram efficiently classifying star-forming, composite, and AGN galaxies.

- We analyzed the galaxy evolution using the three main BPT diagrams: [N II $] / \mathrm{H} \alpha$, [S II] $/ \mathrm{H} \alpha$, and [O I] $\lambda 6300 / \mathrm{H} \alpha$ vs. [O III] $\lambda 5007 / \mathrm{H} \beta$ in our four redshift bins, observing an evolution toward higher values of the $[\mathrm{O} \mathrm{III}] \lambda 5007 / \mathrm{H} \beta$ ratio. This evolution is a consequence of the metallicity evolution as redshift increases, reflected in the three BPT diagrams, because the ratio [O III] $\lambda 5007 / \mathrm{H} \beta$ is a good metallicity indicator. As a result, a metallicity decrement will be reflected in higher values of this ratio.

- We analyzed the evolution of the $M-Z$ and $L-Z$ relations, observing that at higher redshift values, both relations evolve towards lower values of metallicity. We discovered that the 


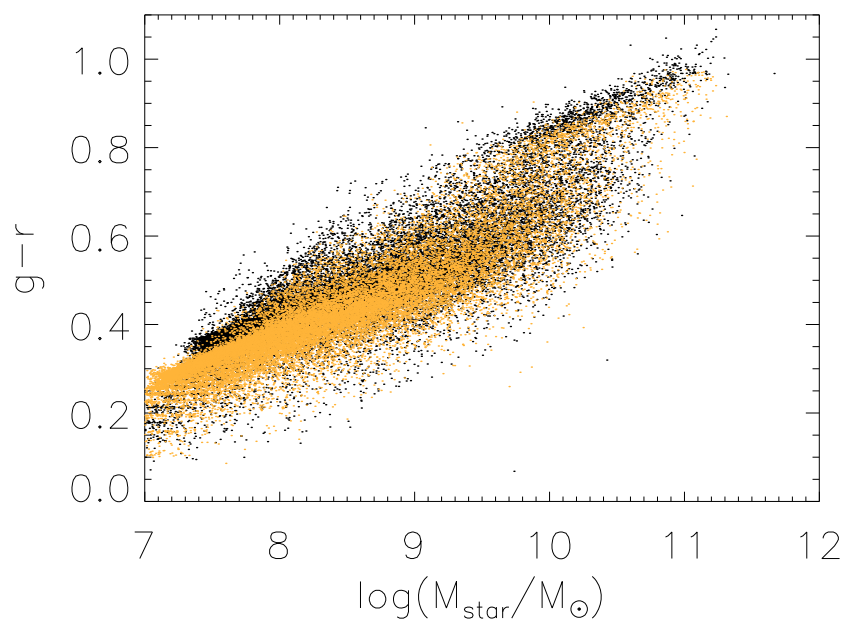

Fig. 15. Mass versus $g-r$ color for Millennium galaxies. Black and yellow dots represent galaxies at $z_{0}$ and $z_{3}$, respectively. (See the electronic edition of the Journal for a color version of this figure.)

flat zone of the $M-Z$ relation reported by Tremonti et al. (2004) for galaxies with $\log \left(M_{\text {star }} / M_{\odot}\right) \gtrsim 10.5$, is mainly constituted by galaxies at $z>0.1$ (samples at $z_{1}, z_{2}$ and $z_{3}$ ). Galaxies at $z_{0}$ redshift could be fitted with a linear function. Our $M-Z$ relation at redshift $z_{3}$ is $\sim 0.2$ dex lower than our local one.

- Our fit to the $M-Z$ relation for sample $z_{3}$ agrees with the one of Erb et al. (2006a) at $z \sim 2.2$. We attribute this similarity to the galaxy morphology in the different samples, since our $z_{3}$ sample conforms to late-type galaxies, while the sample of Erb et al. is composed of a mix of early and late-type galaxies. According to Calura et al. (2009), the $M-Z$ relation of late-type galaxies will have systematically lower metallicities than an $M-Z$ relation composed of a mix of early and late-type galaxies.

- We analyzed the evolution of the mass-to-light ratio, observing lower $M / L$ ratios as redshift increases. For a narrow range of absolute magnitudes in the $z_{3}$ sample, we have a wide range of mass, making it possible to generate the $M-Z$ relation, but difficult to generate the $L-Z$ relation.

- The decrement in metallicity observed in previous papers for galaxies at redshift $z_{3}$ (Lara-López et al. 2009a,b) is also observed, even though in this study we are not restricting our galaxy luminosities as in our previous studies.

- We estimated the SFR and SSFR for our sample of galaxies and analyzed its relation with $12+\log (\mathrm{O} / \mathrm{H})$ and $\log \left(M_{\text {star }} / M_{\odot}\right)$, confirming the existence of the MS reported by Noeske et al. (2007) in the $\log (\mathrm{SFR})$ vs. $\log \left(M_{\text {star }} / M_{\odot}\right)$ plot. Consistently, we found that higher SFRs and SSFRs increase with redshift.

- We analyzed the morphology of our galaxies through the $g-r$ color, the concentration index $R_{90} / R_{50}$, and the SSFR, concluding that the best method of determining the morphology was by combining both a color of $g-r<0.6$ and a $\log (S S F R)>10$ for selecting late-type galaxies.

- Our $z_{3}$ sample of galaxies is mainly formed by late-type galaxies, which helped us to classify morphological types at lower redshift. That at higher redshift the fraction of latetype galaxies is greater, was confirmed by using mock galaxy catalogs from Millennium simulations.

- At the higher redshift, we found a population with higher SFR and SSFR than the galaxies in the $z_{0}$ sample. After classifying late and early-type galaxies in the $z_{0}$ sample, we realized that the observed tail showing higher SFR and SSFR is formed by late-type galaxies, demonstrating the connection of the galaxy morphology with the SFR in a new fashion.

Our work provides a useful tool for classifying galaxies with the S2N2 diagrams, and for demonstrating how galaxies evolve on the BPT diagrams as a consequence of metallicity evolution. We also analyzed the mass, metallicity, and SFR relations, noting that galaxies in the redshift sample $z_{3}$ have lower values of metallicity, higher SFRs, and morphology indicators associated to late types. In this study we pointed out the importance of the morphology of galaxies when deriving conclusions, since a sample composed of late-type galaxies will show lower values of metallicity than ones formed by a mix of morphological types.

Acknowledgements. This work was supported by the Spanish Plan Nacional de Astronomía y Astrofísica under grant AYA2008-06311-C02-01. The Sloan Digital Sky Survey (SDSS) is a joint project of The University of Chicago, Fermilab, the Institute for Advanced Study, the Japan Participation Group, The Johns Hopkins University, the Max-Planck-Institute for Astronomy, Princeton University, the United States Naval Observatory, and the University of Washington. Apache Point Observatory, site of the SDSS, is operated by the Astrophysical Research Consortium. Funding for the project has been provided by the Alfred P. Sloan Foundation, the SDSS member institutions, the National Aeronautics and Space Administration, the National Science Foundation, the US Department of Energy, and Monbusho. The official SDSS web site is www.sdss.org. The Millennium Simulation databases used in this paper and the web application providing online access to them were constructed as part of the activities of the German Astrophysical Virtual Observatory. We thank the Starlight Project Team (UFSC, Brazil), especially William Schoenell, who helped us downloads the whole data set. We thank Romano Corradi for giving us the idea of using the S2N2 diagram in galaxies. We thank Kerttu Viironen for providing us the lines and metallicity data for several $\mathrm{H}$ II and PNe to test the efficiency of the S2N2 diagram. We also thank the anonymous referee for his/her constructive comments. Maritza A. Lara-López is supported by a CONACyT and SEP Mexican fellowships.

\section{References}

Abraham, R. G., van den Bergh, S., \& Nair, P. 2003, ApJ, 588, 218 Adelman-McCarthy, J. K., Agüeros, M. A., Allam, S. S., et al. 2007, ApJS, 172, 634

Asari, N. V., Cid Fernandes, R., Stasińska, G., et al. 2007, MNRAS, 381, 263 Baldwin, J., Phillips, M., \& Terlevich, R. 1981, PASP, 93, 5 (BPT)

Baldry, I. K., Glazebrook, K., Brinkmann, J., et al. 2004, ApJ, 600, 681

Bauer, A. E., Drory, N., Hill, G. J., \& Feulner, G. 2005, ApJ, 621, L89 Bell, E. F., Papovich, C., Wolf, C., et al. 2005, ApJ, 625, 23

Bower, R. G., Benson, A. J., Malbon, R., et al. 2006, MNRAS, 370, 645 Brinchmann, J., \& Ellis, R. S. 2000, ApJ, 536, L77

Brinchmann, J., Charlot, S., White, S. D. M., et al. 2004, MNRAS, 351, 1151

Brodie, J. P., \& Huchra, J. P. 1991, ApJ, 379, 157

Brooks, A. M., Governato, F., Booth, C. M., et al. 2007, ApJ, 655, L17

Bruzual, G., \& Charlot, S. 2003, MNRAS, 344, 1000

Buat, V., Boissier, S., Burgarella, D., et al. 2008, A\&A, 483, 107

Calura, F., Pipino, A., Chiappini, C., Matteucci, F., \& Maiolino, R. 2009, A\&A, 504,373

Cantó, J. 1981, in Investigating the Universe, ed. Z. Kopal, \& F. D. Kahn (Dordrecht: Reidel), 95

Caputi, K. I., Dole, H., Lagache, G., et al. 2006, ApJ, 637, 727

Cardelli, J. A., Clayton, G. C., \& Mathis, J. S. 1989, ApJ, 345, 245

Carollo, C. M., \& Lilly, S. J. 2001, ApJ, 548, L153

Chabrier, G. 2003, PASP, 115, 763

Charlot, S., Kauffmann, G., Longhetti, M., et al. 2002, MNRAS, 330, 876

Cid Fernandes, R., Mateus, A., Sodré, L., Stasińska, G., \& Gomes, J. M. 2005, MNRAS, 358, 363

Cid Fernandes, R., Asari, N. V., Sodré, L., et al. 2007, MNRAS, 375, L16

Cohen, J. G. 1976, ApJ, 203, 587

Condon, J. J., Cotton, W. D., \& Broderick, J. J. 2002, AJ, 124, 675

Conselice, C. J. 2006, MNRAS, 373, 1389

Cowie, L. L., Songaila, A., Hu, E. M., \& Cohen, J. G. 1996, AJ, 112, 839

De Lucia, G., Kauffmann, G., \& White, S. D. M. 2004, MNRAS, 349, 1101

Dekel, A., \& Silk, J. 1986, ApJ, 303, 39

Deng, X., He, J., Wu, P., \& Ding, Y. 2009, ApJ, 699, 948 
M. A. Lara-López et al.: Evolution from fundamental parameters in SDSS galaxies. II.

Denicoló, G., Terlevich, R., \& Terlevich, E. 2002, MNRAS, 330, 69

Dickinson, M., Stern, D., Giavalisco, M., et al. 2004, ApJ, 600, L99

Donas, J., \& Deharveng, J. M. 1984, A\&A, 140, 325

Dopita, M. A., Kewley, L. J., Heisler, C. A., \& Sutherland, R. S. 2000, ApJ, 542, 224

Dopita, M. A., Periera, L., Kewley, L. J., \& Capacciolo, M. 2002, ApJS, 143, 47 Dopita, M. A., Fischera, J., Sutherland, R. S., et al. 2006, ApJS, 167, 177 Efstathiou, G. 2000, MNRAS, 317, 697

Ellison, S. L., Patton, D. R., Simard, L., \& McConnachie, A. W. 2008, ApJ, 672, L107

Erb, D. K., Shapley, A. E., Pettini, M., et al. 2006a, ApJ, 644, 813

Erb, D. K., Steidel, C. C., Shapley, A. E., et al. 2006b, ApJ, 646, 107

Faber, S. M., Willmer, C. N. A., Wolf, C., et al. 2007, ApJ, 665, 265

Feulner, G., Gabasch, A., Salvato, M., et al. 2005, ApJ, 633, L9

Finlator, K., \& Davé, R. 2008, MNRAS, 385, 2181

Fioc, M., \& Rocca-Volmerange, B. 1997, A\&A, 329, 950

Gallagher, J. S., Hunter, D. A., \& Bushouse, H. 1989, AJ, 97, 700

García-Lario, O., Manchado, A., Riera, A., Mampaso, A., \& Pottasch, S. R. 1991, A\&A, 249, 223

Garnett, D. R., Shields, G. A., Skillman, E. D., Sagan, S. P., \& Dufour, R. J. 1997, ApJ, 489, 36

Gavazzi, G., \& Scodeggio, M. 1996, A\&A, 312, L29

Gavazzi, G., Bonfanti, C., Sanvito, G., Boselli, A., \& Scodeggio, M. 2002, ApJ, 576,135

Goto, T., Okamura, S., McKay, T. A., et al. 2002, PASJ, 54, 515

Gunn, J. E., Siegmund, W. A., Mannery, E. J., et al. 2006, AJ, 131, 2332

Guzmán, R., Gallego, J., Koo, D. C., et al. 1997, ApJ, 489, 559

Harper, D. A., \& Low, F. J. 1973, ApJ, 182, L89

Hammer, F., Flores, H., Elbaz, D., et al. 2005, A\&A, 430, 115

Hubble, E. 1926, ApJ, 64, 321

Jansen, R. A., Franx, M., \& Fabricant, D. 2001, ApJ, 551, 825

Juneau, S., Glazebrook, K., Crampton, D., et al. 2005, ApJ, 619, L135

Kauffmann, G., Heckman, T. M., Tremonti, C., et al. 2003a, MNRAS, 346, 1055 (Kauf03)

Kauffmann, G., Heckman, T. M., White, S. D., et al. 2003b, MNRAS, 341, 54

Kauffmann, G., White, S. D. M., Heckman, T. M., et al. 2004, MNRAS, 353, 713

Kennicutt, R. C. 1998, ARA\&A, 36, 189

Kennicutt, R. C., \& Kent, S. M. 1983, AJ, 88, 1094

Kennicutt, R. C., Jr., Tamblin, P., \& Congdon, C. 1994, 435, 22

Kewley, L. J., \& Dopita, M. A. 2002, ApJS, 142, 35

Kewley, L. J., \& Ellison, S. L. 2008, ApJ, 681, 1183

Kewley, L. J., Dopita, M. A., Sutherland, R. S., Heisler, C. A., \& Trevena, J. 2001, ApJ, 556, 121 (Kew01)

Kewley, L. J., Geller, M. J., \& Jansen, R. A. 2004, AJ, 127, 2002

Kewley, L. J., Jansen, R. A., \& Geller, M. J. 2005, PASP, 117, 227

Kewley, L. J., Groves, B., Kauffmann, G., \& Heckman, T. 2006, MNRAS, 372, 961

Kobayashi, C., Springel, V., \& White, S. D. M. 2007, MNRAS, 376, 1465

Kobulnicky, H. A., \& Kewley, L. J. 2004, ApJ, 617, 204

Köppen, J., Weidner, C., \& Kroupa, P. 2007, MNRAS, 375, 673

Kroupa, P. 2001, MNRAS, 322, 231

Lamareille, F., Brinchmann, J., Contini, T., et al. 2009, A\&A, 495, 53

Lara-López, M. A., Cepa, J., Bongiovanni, A., et al. 2009a, A\&A, 493, L5

Lara-López, M. A., Cepa, J., Bongiovanni, A., et al. 2009b, A\&A, 505, 529

Lara-López, M. A., Cepa, J., Bongiovanni, A., et al. 2010, A\&A, submitted [arXiv: 1005.0509]

Larson, R. B. 1974, MNRAS, 169, 229

Larson, R. B., \& Tinsley, B. M. 1978, ApJ, 219, 46

Lee, J. C. 2006, Ph.D. Thesis, Univ. Arizona

Lee, J. H., Lee, M. G., Kim, T., et al. 2007a, ApJ, 663, L69

Lee, J. C., Kennicutt, R. C., Funes, S. J., et al. 2007b, ApJ, 671, L113

Leitherer, C., Schaerer, D., Goldader, J. D., et al. 1999, ApJS, 123, 3

Lequeux, J., Peimbert, M., Rayo, J. F., Serrano, A., \& Torres-Peimbert, S. 1979, A\&A, 80, 155

Levesque, E. M., Kewley, L. J., \& Larson, K. L. 2010, ApJ, 139, 712

Liang, Y. C., Yin, S. Y., Hammer, F., et al. 2006, ApJ, 652, 257

Lilly, S. J., Carollo, C. M., \& Stockton, A. 2003, ApJ, 597, 730

Lintott, C. J., Schawinski, K., Slosar, A., et al. 2008, MNRAS, 389, 1179

López-Sánchez, A. R. 2010, A\&A, accepted [arXiv: 1005. 0659]

López-Sánchez, A. R., \& Esteban, C. 2010, A\&A, 517, A85

MacLow, M., \& Ferrara, A. 1999, ApJ, 513, 142

Magrini, L., Perinotto, M., Corradi, R. L. M., \& Mampaso, A. 2003, A\&A, 400, 511

Maiolino, R., Nagao, T., Grazian, A., et al. 2008, A\&A, 488, 463
Maier, C., Meisenheimer, K., \& Hippelein, H. 2004, A\&A, 418, 475

Maier, C., Lilly, S., Carollo, C. M., Stockton, A., \& Brodwin, M. 2005, ApJ, 634, 849

Masters, K. L., Mosleh, M., Romer, A. K., et al. 2010, MNRAS, 405, 783

Mateus, A., Sodré, L., Cid Fernandes, R., et al. 2006, MNRAS, 370, 721

McCall, M. L., Rybski, P. M., \& Shields, G. A. 1985, ApJS, 57, 1

Moustakas, J., \& Kennicutt, R. C. Jr. 2006, ApJ, 651, 155

Mouhcine, M., Gibson, B. K., Renda, A., \& Kawata, D. 2008, A\&A, 486, 711

Nakamura, O., Fukugita, M., Yasuda, N., et al. 2003, AJ, 125, 1682

Noeske, K. G., Weiner, B. J., Faber, S. M., et al. 2007a, ApJ, 660, L43

Noeske, K. G., Faber, S. M., Weiner, B. J., et al. 2007b, ApJ, 660, L47

Osterbrock, D. R. 1989, Astrophysics of Gaseous Nebulae and Active Galactic Nuclei (Mill Valley CA: University Science Books)

Pagel, B. E. J., Edmunds, M. G., Blackwell, D. E., Chun, M. S., \& Smith, G. 1979, MNRAS, 189, 95

Pannella, M., Carilli, C. L., Daddi, E., et al. 2009, ApJ, 698, L116

Papovich, C., Moustakas, L. A., Dickinson, M., et al. 2006, ApJ, 640, 92

Park, C., \& Choi, Y. 2005, ApJ, 635, L29

Pérez-Montero, E., \& Contini, T. 2009, MNRAS, 398, 949

Pérez-González, P. G., Rieke, G. H., Egami, E., et al. 2005, ApJ, 630, 82

Pettini, M., \& Pagel, B. E. J. 2004, MNRAS, 348, L59

Pilyugin, L. S., \& Ferrini, F. 2000, A\&A, 358, 72

Raimann, D., Storchi-Bergmann, T., Bica, E., Melnick, J., \& Schmitt, H. 2000, MNRAS, 316, 559

Reddy, N. A., Steidel, C. C., Fadda, D., et al. 2006, ApJ, 644, 792

Richer, M. G., \& McCall, M. L. 1995, ApJ, 445, 642

Rieke, G. H., \& Lebofsky, M. J. 1978, ApJ, 220, L37

Riesgo, H., \& López, J. A. 2005, Rev. Mex. Astron. Astrofis., 41, 57

Riesgo, H., \& López, J. A. 2006, Rev. Mex. Astron. Astrofis., 42, 47

Roberts, M. S. 1963, ARA\&A, 1, 149

Rodrigues, M., Hammer, F., Flores, H., et al. 2008, A\&A, 492, 371

Rosa-González, D., Terlevich, E., \& Terlevich, R. 2002, MNRAS, 332, 283

Rosa González, D., Terlevich, E., Jiménez Bailón, E., et al. 2009, MNRAS, 399, 487

Rovilos, E., Georgantopoulos, I., Tzanavaris, P., et al. 2009, A\&A, 502, 85

Savaglio, S., Glazebrook, K., Le Borgne, D., et al. 2005, ApJ, 635, 260

Sabbadin, F., Minello, S., \& Bianchini, A. 1977, A\&A, 60, 174

Salim, S., Charlot, S., Rich, M., et al. 2005, ApJ, 619, L39

Salim, S., Dickinson, M., Rich, R. M., et al. 2009, ApJ, 700, 161

Sandage, A. R. 1961, The Hubble Atlas of Galaxies, Carnegie Institute of Washington, Washington

Scannapieco, C., Tissera, P. B., White, S. D. M., \& Springel, V. 2008, MNRAS, 389,1137

Scarlata, C., Carollo, C. M., Lilly, S., et al. 2007, ApJS, 172, 406

Schawinski, K., Virani, S., Simmons, B., et al. 2009, ApJ, 692, L19

Searle, L., Sargent, W. L. W., \& Bagnuolo, W. G. 1973, ApJ, 179, 427

Serjeant, S., Gruppioni, C., \& Oliver, S. 2002, MNRAS, 330, 621

Shapley, A. E., Steidel, C. C., Erb, D. K., et al. 2005, ApJ, 626, 698

Shimasaku, K., Fukugita, M., Doi, M., et al. 2001, AJ, 122, 1238

Skillman, E. D., Kennicutt, R. C., Jr., \& Hodge, P. W. 1989, ApJ, 347, 875

Springel, V., White, S. D. M., Jenkins, A., et al. 2005, Nature, 435, 629

Stasińska, G., Cid Fernandes, R., Mateus, A., Sodré, L., \& Asari, N. V. 2006, MNRAS, 371, 972

Stoughton, C., Lupton, R. H., Bernardi, M., et al. 2002, AJ, 123, 485

Strauss, M. A., Weinberg, D. H., Lupton, R. H., et al. 2002, AJ, 124, 1810

Strateva, I., Ivezić, Z., Knapp, G. R., et al. 2001, AJ, 122, 1861

Tassis, K., Kravtsov, A. V., \& Gnedin, N. Y. 2008, ApJ, 672, 888

Telesco, C. M., \& Harper, D. A. 1980, ApJ, 235, 392

Tinsley, B. M. 1968, ApJ, 151, 547

Tinsley, B. M. 1972, A\&A, 20, 383

Tremonti, C. A., Heckman, T. M., Kauffmann, G., et al. 2004, ApJ, 613, 898 (T04)

Tresse, L., \& Maddox, S. J. 1998, ApJ, 495, 691

Vale Asari, N., Cid Fernandes, R., Gomes, J. M., et al. 2009, MNRAS, 396, L71

de Vaucouleurs, G., de Vaucouleurs, A., Corwin, H. G., et al. 1991, Third

Reference Catalog of Bright Galaxies (New York: Springer-Verlag)

Veilleux, S., \& Osterbrock, D. E. 1987, ApJS, 63, 295

Viironen, K., Delgado-Inglada, G., Mampaso, A., Magrini, L., \& Corradi, R. L. M. 2007, MNRAS, 381, 1719

Wang, Y., Yang, X., Mo, H. J., \& van den Bosch, F. C. 2007, ApJ, 664, 608

Weinmann, A. M., van den Bosch, F. C., Yang, X., \& Mo, H. J. 2006, MNRAS, 366,2

Wolf, C., Aragón-Salamanca, A., \& Balogh, M. 2009, MNRAS, 393, 1302

Yamauchi, C., \& Goto, T. 2005, MNRAS, 359, 1557

Zaritsky, D., Kennicutt, R. C., \& Huchra, J. P. 1994, ApJ, 420, 87 